TRANSACTIONS OF THE

AMERICAN MATHEMATICAL SOCIETY

Volume 362, Number 1, January 2010, Pages 509-535

S 0002-9947(09)04938-1

Article electronically published on August 7, 2009

\title{
NONVANISHING VECTOR FIELDS ON ORBIFOLDS
}

\author{
CARLA FARSI AND CHRISTOPHER SEATON
}

\begin{abstract}
We introduce a complete obstruction to the existence of nonvanishing vector fields on a closed orbifold $Q$. Motivated by the inertia orbifold, the space of multi-sectors, and the generalized orbifold Euler characteristics, we construct for each finitely generated group $\Gamma$ an orbifold called the space of $\Gamma$-sectors of $Q$. The obstruction occurs as the Euler-Satake characteristics of the $\Gamma$-sectors for an appropriate choice of $\Gamma$; in the case that $Q$ is oriented, this obstruction is expressed as a cohomology class, the $\Gamma$-Euler-Satake class. We also acquire a complete obstruction in the case that $Q$ is compact with boundary and in the case that $Q$ is an open suborbifold of a closed orbifold.
\end{abstract}

\section{INTRODUCTION}

If $M$ is a closed manifold, then it is well known that $M$ admits a smooth, nonvanishing vector field if and only if the Euler characteristic of $M$ vanishes (see e.g. [6]). For the case of a closed orbifold $Q$, the fact that the existence of a nonvanishing vector field ensures the vanishing of the Euler-Satake characteristic (i.e. Satake's Euler characteristic as a $V$-manifold) is a trivial consequence of Satake's Poincaré-Hopf Theorem in [15. In [16, Corollary 3.4], the second author offered a different Poincaré-Hopf theorem, demonstrating that a nonvanishing vector field also implies that the Euler characteristic of the underlying topological space of $Q$ vanishes. However, the converse of both of these statements is false; it is easy to construct examples of orbifolds such that both characteristics vanish, yet whose singular strata force any vector field to vanish (see [17]).

Similarly, if $M$ is an open manifold or manifold with boundary, then it is well known that $M$ always admits a nonvanishing vector field. We note that no requirements are made of the behavior of the vector field on the boundary; i.e. it need not be tangent to the boundary or pointing in or out of $M$. The case of orbifolds is again not as straightforward, however, as closed components of the singular strata may force a vector field to vanish.

In 17, the second author introduced a complete cohomological obstruction to the existence of nonvanishing vector fields on closed orbifolds with cyclic local groups. In this case, the obstruction was an element of the Chen-Ruan orbifold cohomology (see [4] or 1]), which is additively the cohomology of the inertia orbifold. For cyclic orbifolds, the cohomology of the inertia orbifold is large enough to produce

Received by the editors August 12, 2008.

2000 Mathematics Subject Classification. Primary 22A22, 57R25; Secondary 55S91, 58H05.

Key words and phrases. Orbifold, orbifold with boundary, vector field, orbifold Euler characteristic, orbifold Euler class, orbifold sector.

The second author was partially supported by a Rhodes College Faculty Development Endowment Grant.

(c)2009 American Mathematical Society 
a complete obstruction. Here, we generalize the construction of the inertia orbifold to introduce the space of $\Gamma$-sectors of a general orbifold $Q$. Roughly speaking, the inertia orbifold is the set of pairs $(x,(g))$ where $x$ is an object in an orbifold groupoid $\mathcal{G}$ presenting $Q$ and $(g)$ is the conjugacy class of an element $g$ in the isotropy group of $x$. Hence, $(g)$ can be thought of as the conjugacy class of a homomorphism from $\mathbb{Z}$ into the isotropy group of $x$. In contrast, the space of $\Gamma$ sectors is constructed by choosing homomorphisms from a fixed, finitely generated group $\Gamma$ into the isotropy group. The orbifold structure of the space of $\Gamma$-sectors is given by a translation groupoid via an action of the orbifold groupoid $\mathcal{G}$. When $\Gamma$ is chosen appropriately, the set of Euler-Satake characteristics of these $\Gamma$-sectors acts as a complete obstruction to the existence of nonvanishing vector fields on $Q$. When $Q$ is oriented, we define in the cohomology of the space of $\Gamma$-sectors an Euler class $e_{\Gamma}^{E S}(Q)$, called the $\Gamma$-Euler-Satake class of $Q$, that contains this information. We demonstrate the following.

Theorem 1.1. Let $Q$ be a closed orbifold and $\Gamma$ a finitely generated group that covers the local groups of $Q$. Then $Q$ admits a smooth, nonvanishing vector field if and only if $\chi_{E S}\left(\tilde{Q}_{(\phi)}\right)=0$ for each $\Gamma$-sector $\tilde{Q}_{(\phi)}$. In the case that $Q$ is oriented, this is equivalent to $e_{\Gamma}^{E S}(Q)=0$.

We will also prove Theorems 4.7 and 4.11, which give similar results in the cases where $Q$ is a compact orbifold with boundary and an open suborbifold of a closed orbifold, respectively.

The construction of the $\Gamma$-sectors is motivated by a construction of Tamanoi in 19 and 20 (see also 2 and 11) which was used to define a generalized orbifold Euler characteristic of a global quotient orbifold, i.e. an orbifold that admits a presentation as $M / G$ where $M$ is a smooth manifold and $G$ is a finite group acting smoothly. Using the techniques of [4] and 1, we produce a similar construction for orbifolds that do not admit such a presentation.

Late in the preparation of this paper, the authors became aware of a similar construction in [7, pages 4-8]. Leida notes (on page 14) that his space of fixedpoint sectors can be identified with the mapping space of faithful homomorphisms from finite groups into the orbifold groupoid $\mathcal{G}$; we take this approach using a fixed, not necessarily finite, group, and do not require that the homomorphisms be faithful. It is clear that a similar obstruction theorem can be proven using Leida's construction. However, the construction contained here relates specifically to and generalizes existing constructions for quotients, including orbifold Euler characteristics. In a forthcoming paper, the authors will explore the relationship between the $\Gamma$-sectors given here and other constructions, including the inertia orbifold, the space of multi-sectors in [4] and 1], and orbifold Euler characteristics. Here, our focus is on the properties of the construction itself and the obstruction to nonvanishing vector fields.

The outline of this paper is as follows. In Section 2, we give the construction of the $\Gamma$-sectors as well as the definition of the class $e_{\Gamma}^{E S}(Q)$. In Section 3, we determine the topological properties of the $\Gamma$-sectors that we will require. In Section 4 , we prove Theorem 1.1. We also prove, as Theorems 4.7 and 4.11, analogous results in the cases of $Q$ compact with boundary and $Q$ an open suborbifold of a closed orbifold, respectively. 
Note that many authors require of an orbifold $Q$ that each local group $G_{x}$ act within a chart with a fixed-point set of codimension at least 2 . We make this requirement as well. However, we note that the construction of the $\Gamma$-sectors in Subsections 2.1 and 2.2 does not require this hypothesis.

\section{Definitions}

2.1. The $\Gamma$-sectors of an orbifold. We assume throughout that $Q$ is an $n$ dimensional orbifold; we do not assume that $Q$ is effective or that it admits a presentation as quotient. We use the definition and notation in [1] (see also [3], 8], [9], and [14 for background information). Recall that a presentation of $Q$ is given by an orbifold groupoid $\mathcal{G}$ (a proper étale Lie groupoid) and a homeomorphism between the orbit space $|\mathcal{G}|$ of $\mathcal{G}$ and the underlying space of $Q$ (see [1, pages 19-23]). We take a fixed orbifold groupoid $\mathcal{G}$ and identify the underlying space of $Q$ with $|\mathcal{G}|$. Let $\sigma: G_{0} \rightarrow|\mathcal{G}|=Q$ denote the quotient map. As usual, $G_{0}$ and $G_{1}$ denote the spaces of objects and arrows, respectively, in $\mathcal{G}, s$ and $t$ denote the source and target maps, respectively, and $G_{x}$ denotes the set of loops at a point $x \in G_{0}$, the isotropy group of $x$.

Let $p \in Q$ correspond to the $\mathcal{G}$-orbit of $x \in G_{0}$ so that $\sigma(x)=p$. There is a neighborhood $V_{x}$ of $x$ in $G_{0}$ which is diffeomorphic to $\mathbb{R}^{n}$ in such a way that $x$ corresponds to the origin and the $\left.\mathcal{G}\right|_{V_{x}}=(s, t)^{-1}\left(V_{x} \times V_{x}\right)$-action corresponds to a linear $G_{x}$-representation (see [1, page 19 and Proposition 1.44, page 21], [8, page 8], or [9, page 15]). For ease of notation, we will identify $V_{x}$ with a subset of $\mathbb{R}^{n}$ without explicit reference to a choice of diffeomorphism. In this context, we use $\pi_{x}: V_{x} \rightarrow Q$ to denote the restriction of the quotient map $\sigma$ to $V_{x}$ and use $U_{p}$ to denote $\pi_{x}\left(V_{x}\right) \subseteq Q$. Then we call $\left\{V_{x}, G_{x}, \pi_{x}\right\}$ a linear orbifold chart for $Q$ at $x$. Whenever we use this notation, we will assume that the chart has these properties. In particular, such a chart defines a groupoid homomorphism $\xi_{x}:\left.\mathcal{G}\right|_{V_{x}} \rightarrow G_{x}$ where the group $G_{x}$ is treated as a groupoid with space of objects $\{x\}$, identifying $\left.\mathcal{G}\right|_{V_{x}}$ with $G_{x} \ltimes V_{x}$. As $G_{x}$ acts on $V_{x}$, for each $y \in V_{x}$, $\xi_{x}$ defines a bijection between $s^{-1}(y) \cap t^{-1}\left(V_{x}\right)$ and $G_{x}$. In particular, $\xi_{x}$ restricts to an injective group homomorphism denoted by $\xi_{x}^{y}=\left.\left(\xi_{x}\right)\right|_{G_{y}}: G_{y} \rightarrow G_{x}$.

If $x^{\prime} \in G_{0}$ is another point in the orbit of $x$ such that $\sigma\left(x^{\prime}\right)=p$, then there is a $g \in G_{1}$ with $s(g)=x, t(g)=x^{\prime}$. By shrinking $V_{x}$ if necessary, we can assume that $s$ restricts to a diffeomorphism $s_{g}$ from a neighborhood of $g$ to $V_{x}$. Then $g G_{x} g^{-1}=G_{x^{\prime}}$ so that $G_{x}$ and $G_{x^{\prime}}$ are isomorphic, and $t \circ s_{g}^{-1}\left(V_{x}\right)$ is a neighborhood of $x^{\prime}$ in $G_{0}$ diffeomorphic to $\mathbb{R}^{n}$ where $x^{\prime}$ corresponds to the origin; hence, $g$ induces an isomorphism of orbifold charts, and $\pi_{x^{\prime}} \circ t \circ s_{g}^{-1}=\pi_{x}$. Hence, we may refer to the isotropy group of a point $p \in Q$. By this, we of course mean the isotropy group of an $x \in G_{0}$ with $\sigma(x)=p$, which (up to isomorphism) does not depend on the choice of $x$.

The following definition follows [1, pages 52-4].

Definition 2.1 (Space of objects of the $\Gamma$-sectors). Let $Q$ be an orbifold and $\Gamma$ a finitely generated group. We let $\mathcal{S}_{\mathcal{G}}^{\Gamma}$ denote the set

$$
\mathcal{S}_{\mathcal{G}}^{\Gamma}=\bigcup_{x \in G_{0}} \operatorname{HOM}\left(\Gamma, G_{x}\right) .
$$

A point in $\mathcal{S}_{\mathcal{G}}^{\Gamma}$ will be denoted by $\phi_{x}$ where $\phi_{x} \in \operatorname{HOM}\left(\Gamma, G_{x}\right)$. We let $\beta_{\Gamma}: \mathcal{S}_{\mathcal{G}}^{\Gamma} \rightarrow G_{0}$ denote the map $\beta_{\Gamma}\left(\phi_{x}\right)=x$. 
For each $\phi_{x} \in \mathcal{S}_{\mathcal{G}}^{\Gamma}$, pick a linear chart $\left\{V_{x}, G_{x}, \pi_{x}\right\}$ for $Q$ at $x \in G_{0}$. Let $V_{x}^{\left\langle\phi_{x}\right\rangle}=$ $\bigcap_{\gamma \in \Gamma} V_{x}^{\phi_{x}(\gamma)}$ denote the fixed-point subset of $\operatorname{Im} \phi_{x}$. Let the map

$$
\kappa_{\phi_{x}}: V_{x}^{\left\langle\phi_{x}\right\rangle} \longrightarrow \mathcal{S}_{\mathcal{G}}^{\Gamma}
$$

be defined as follows. For each $y \in V_{x}, \xi_{x}^{y}: G_{y} \rightarrow G_{x}$ is an injective group homomorphism. If $y \in V_{x}^{\left\langle\phi_{x}\right\rangle}$, we have $\operatorname{Im} \phi_{x} \leq \xi_{x}^{y}\left(G_{y}\right) \leq G_{x}$, so that we can define $\phi_{y}:=\left(\xi_{x}^{y}\right)^{-1} \circ \phi_{x}: \Gamma \rightarrow G_{y}$. Let $\kappa_{\phi_{x}}(y)=\phi_{y}$.

Lemma 2.2. Let $Q$ be an orbifold and let $\Gamma$ be a finitely generated group. The $\left\{V_{x}^{\left\langle\phi_{x}\right\rangle}, \kappa_{\phi_{x}}\right\}$ give $\mathcal{S}_{\mathcal{G}}^{\Gamma}$ the structure of a smooth manifold (with connected components of different dimensions) in such a way that $\beta_{\Gamma}$ is a smooth surjective map.

Proof. Fix $\phi_{x} \in \mathcal{S}_{\mathcal{G}}^{\Gamma}$. It is clear that $\kappa_{\phi_{x}}$ is injective, as it is inverted on its image by $\beta_{\Gamma}$. We give $\mathcal{S}_{\mathcal{G}}^{\Gamma}$ the topology induced by the $\kappa_{\phi_{x}}$. Then the $\left\{V_{x}^{\left\langle\phi_{x}\right\rangle}, \kappa_{\phi_{x}}\right\}$ define manifold charts at each point $\phi_{x} \in \mathcal{S}_{\mathcal{G}}^{\Gamma}$. If a different linear chart is chosen at $x$, then it clearly yields an equivalent (manifold) chart for $\mathcal{S}_{\mathcal{G}}^{\Gamma}$. If $\phi_{x}, \psi_{y} \in \mathcal{S}_{\mathcal{G}}^{\Gamma}$ such that $\kappa_{\phi_{x}}\left(V_{x}^{\left\langle\phi_{x}\right\rangle}\right) \cap \kappa_{\psi_{y}}\left(V_{x}^{\left\langle\psi_{y}\right\rangle}\right) \neq \emptyset$, then

$$
\kappa_{\phi_{x}}^{-1} \circ \kappa_{\phi_{y}}: V_{y}^{\left\langle\phi_{y}\right\rangle} \longrightarrow V_{x}^{\left\langle\phi_{x}\right\rangle}
$$

is a restriction of the associated transition map for the smooth manifold $G_{0}$ to a submanifold and hence is smooth. Therefore, the $\left\{V_{x}^{\left\langle\phi_{x}\right\rangle}, \kappa_{\phi_{x}}\right\}$ and their finite intersections define an atlas of smooth charts for $\mathcal{S}_{\mathcal{G}}^{\Gamma}$.

For each $\phi_{x} \in \mathcal{S}_{\mathcal{G}}^{\Gamma}$, the map $\beta_{\Gamma} \circ \kappa_{\phi_{x}}$ is the identity on $V_{x}^{\left\langle\phi_{x}\right\rangle}$. Hence $\beta_{\Gamma}$ is smooth.

We define a $\mathcal{G}$-action on $\mathcal{S}_{\mathcal{G}}^{\Gamma}$ by letting $g \in G_{1}$ act via pointwise conjugation. In other words, if $x=s(g)$, for each $\gamma \in \Gamma$ we set

$$
\left(g \phi_{x}\right)(\gamma)=g\left(\phi_{x}(\gamma)\right) g^{-1}
$$

Note that $\beta_{\Gamma}$ is the anchor map of this action and that $g: \beta_{\Gamma}^{-1}(s(g)) \rightarrow \beta_{\Gamma}^{-1}(t(g))$ as each $g\left(\phi_{x}(\gamma)\right) g^{-1}$ is in the isotropy group of $t(g)$. The properties of a groupoid action follow trivially. We let $\mathcal{G}^{\Gamma}$ denote the action groupoid $\mathcal{G} \ltimes \mathcal{S}_{\mathcal{G}}^{\Gamma}$.

As $\mathcal{G}^{\Gamma}$ is the action groupoid for a smooth orbifold groupoid acting on a smooth manifold, $\mathcal{G}^{\Gamma}$ is an orbifold groupoid. Moreover, the anchor map extends to a homomorphism $\beta_{\Gamma}: \mathcal{G}^{\Gamma} \rightarrow \mathcal{G}$ (see [1, pages 39-40]).

Definition 2.3 (Space of $\Gamma$-sectors of $Q$ ). We let $\tilde{Q}_{\Gamma}$ denote the orbit space of $\left|\mathcal{G}^{\Gamma}\right|$ with the orbifold structure given by $\mathcal{G}^{\Gamma}$. We call $\tilde{Q}_{\Gamma}$ the space of $\Gamma$-sectors of $Q$. A point in $\tilde{Q}_{\Gamma}$ is the $\mathcal{G}$-orbit of a point $\phi_{x} \in \mathcal{S}_{\mathcal{G}}^{\Gamma}$, denoted by $\mathcal{G} \phi_{x}$.

Fix $x \in G_{0}$ with orbit $\sigma(x)=p \in Q$ and pick a linear chart $\left\{V_{x}, G_{x}, \pi_{x}\right\}$ for $Q$ at $x$. By Lemma 2.2, for each $\phi_{x} \in \mathcal{S}_{\mathcal{G}}^{\Gamma}, \kappa_{\phi_{x}}: V_{x}^{\left\langle\phi_{x}\right\rangle} \rightarrow \mathcal{S}_{\mathcal{G}}^{\Gamma}$ gives a manifold chart for $\mathcal{S}_{\mathcal{G}}^{\Gamma}$ near $\phi_{x}$. We let $C_{G_{x}}\left(\phi_{x}\right)$ denote the centralizer of $\operatorname{Im} \phi_{x}$ in $G_{x}$ and $\pi_{x}^{\phi_{x}}: V_{x}^{\left\langle\phi_{x}\right\rangle} \rightarrow V_{x}^{\left\langle\phi_{x}\right\rangle} / C_{G_{x}}\left(\phi_{x}\right)$ the quotient map.

Given $g \in s^{-1}(x)$, it is clear from equation (2.1) that $g \phi_{x}=\phi_{x}$ if and only if $g \in C_{G_{x}}\left(\phi_{x}\right)$. Hence, the isotropy group of $\phi_{x}$ in the groupoid $\mathcal{G}^{\Gamma}$ is given by 
$C_{G_{x}}\left(\phi_{x}\right)$. Via $\kappa_{\phi_{x}}$, the $\mathcal{G}$-action on $\kappa_{\phi_{x}}\left(V_{x}^{\left\langle\phi_{x}\right\rangle}\right) \subseteq \mathcal{S}_{\mathcal{G}}^{\Gamma}$ corresponds to the $C_{G_{x}}\left(\phi_{x}\right)$ action on $V_{x}^{\left\langle\phi_{x}\right\rangle}$. With this, we have the following.

Lemma 2.4. Let $Q$ be an orbifold and $\Gamma$ a finitely generated group. For each $\phi_{x} \in \mathcal{S}_{\mathcal{G}}^{\Gamma}$, the manifold chart $\left\{V_{x}^{\left\langle\phi_{x}\right\rangle}, \kappa_{\phi_{x}}\right\}$ for $\mathcal{S}_{\mathcal{G}}^{\Gamma}$ near $\phi_{x}$ induces a linear orbifold chart $\left\{V_{x}^{\left\langle\phi_{x}\right\rangle}, C_{G_{x}}\left(\phi_{x}\right), \pi_{x}^{\phi_{x}}\right\}$ for $\tilde{Q}_{\Gamma}$ at $\phi_{x}$. The restriction $\left.\mathcal{G}^{\Gamma}\right|_{\kappa_{\phi_{x}}\left(V_{x}^{\left\langle\phi_{x}\right\rangle}\right)}$ of $\mathcal{G}^{\Gamma}$ to $\kappa_{\phi_{x}}\left(V_{x}^{\left\langle\phi_{x}\right\rangle}\right) \subseteq \mathcal{S}_{\mathcal{G}}^{\Gamma}$ is isomorphic as a groupoid to $C_{G_{x}}\left(\phi_{x}\right) \ltimes V_{x}^{\left\langle\phi_{x}\right\rangle}$.

Note that $\kappa_{\phi_{x}}\left(V_{x}^{\left\langle\phi_{x}\right\rangle}\right) \subseteq \mathcal{S}_{\mathcal{G}}^{\Gamma}$, so strictly speaking we should say that $\left\{\kappa_{\phi_{x}}\left(V_{x}^{\left\langle\phi_{x}\right\rangle}\right), C_{G_{x}}\left(\phi_{x}\right), \pi_{x}^{\phi_{x}}\right\}$ is a linear orbifold chart for $\tilde{Q}_{\Gamma}$. In this case, we will make explicit use of the diffeomorphism $\kappa_{\phi_{x}}$ to avoid confusing $V_{x}^{\left\langle\phi_{x}\right\rangle} \subseteq V_{x} \subseteq G_{0}$ and $\kappa_{\phi_{x}}\left(V_{x}^{\left\langle\phi_{x}\right\rangle}\right) \subseteq \mathcal{S}_{\mathcal{G}}^{\Gamma}$.

The following is stated for the case of multi-sectors in [1, page 53]; see also [8, page 17] and [5].

Lemma 2.5. Let $\mathcal{G}$ be an orbifold groupoid and $\Gamma$ a finitely generated group. A homomorphism of groupoids $\Phi: \mathcal{G} \rightarrow \mathcal{H}$ induces a homomorphism $\Phi_{*}: \mathcal{G}^{\Gamma} \rightarrow \mathcal{H}^{\Gamma}$. If $\Phi$ is a strong equivalence, then $\Phi_{*}$ is a strong equivalence.

Proof. We let $\Phi_{0}$ and $\Phi_{1}$ denote the maps on objects and arrows, respectively, given by the groupoid homomorphism $\Phi$. To avoid confusion with $\mathcal{G}$, we use $\left(\mathcal{G}^{\Gamma}\right)_{1}$ to denote the space of arrows of $\mathcal{G}^{\Gamma}$, and $s_{\mathcal{G}^{\Gamma}}$ and $t_{\mathcal{G}^{\Gamma}}$ to denote, respectively, the source and target maps of $\mathcal{G}^{\Gamma}$. We use similar notation for $\mathcal{H}$ and $\mathcal{H}^{\Gamma}$, where $\mathcal{H}$ has space of objects $H_{0}$, space of arrows $H_{1}$, etc. Note that the spaces of objects of $\mathcal{G}^{\Gamma}$ and $\mathcal{H}^{\Gamma}$ are $\mathcal{S}_{\mathcal{G}}^{\Gamma}$ and $\mathcal{S}_{\mathcal{H}}^{\Gamma}$, respectively.

Every element of $\left(\mathcal{G}^{\Gamma}\right)_{1}$ consists of an arrow $g \in G_{1}$ and an object $\phi_{x} \in \mathcal{S}_{\mathcal{G}}^{\Gamma}$ such that $s(g)=x$. We let $\left(g, \phi_{x}\right)$ denote the corresponding arrow in $\left(\mathcal{G}^{\Gamma}\right)_{1}$, so that $s_{\mathcal{G}^{\Gamma}}\left[\left(g, \phi_{x}\right)\right]=\phi_{x}$ and $t_{\mathcal{G}^{\Gamma}}\left[\left(g, \phi_{x}\right)\right]=g \phi_{x}$. The map $\left(\beta_{\Gamma}\right)_{1}:\left(\mathcal{G}^{\Gamma}\right)_{1} \rightarrow G_{1}$ is given by $\left(g, \phi_{x}\right) \mapsto g$.

For each $x \in G_{0}, \Phi_{1}: G_{1} \rightarrow H_{1}$ restricts to a group homomorphism from $G_{x}$ to $H_{\Phi_{1}(x)}$. We define a groupoid homomorphism $\Phi_{*}: \mathcal{G}^{\Gamma} \rightarrow \mathcal{H}^{\Gamma}$ as follows. First, we define the map on objects,

$$
\begin{aligned}
\Phi_{* 0}: \mathcal{S}_{\mathcal{G}}^{\Gamma} \longrightarrow \mathcal{S}_{\mathcal{H}}^{\Gamma} \\
: \phi_{x} \longmapsto \Phi_{1} \circ \phi_{x} .
\end{aligned}
$$

Then $\Phi_{* 0}\left(\phi_{x}\right): \Gamma \rightarrow H_{\Phi_{0}(x)}$ is a group homomorphism as required. For each $\gamma \in \Gamma$ and $g \in G_{1}$ with $s(g)=x$ we have

$$
\begin{aligned}
\Phi_{* 0}\left(g \phi_{x}\right)(\gamma) & =\Phi_{1}\left[g \phi_{x}(\gamma) g^{-1}\right] \\
& =\Phi_{1}(g) \Phi_{1}\left[\phi_{x}(\gamma)\right] \Phi_{1}\left(g^{-1}\right) \\
& =\Phi_{1}(g)\left[\Phi_{* 0}\left(\phi_{x}\right)(\gamma)\right] .
\end{aligned}
$$

Hence,

$$
\Phi_{* 0}\left(g \phi_{x}\right)=\Phi_{1}(g) \Phi_{* 0}\left(\phi_{x}\right),
$$

so that $\Phi_{* 0}$ is a $\mathcal{G}$ - $\mathcal{H}$-equivariant map via $\Phi_{1}$.

Fixing $\phi_{x} \in \mathcal{S}_{\mathcal{G}}^{\Gamma}$, pick a (manifold) chart $\left\{V_{x}^{\left\langle\phi_{x}\right\rangle}, \kappa_{\phi_{x}}\right\}$ for $\mathcal{S}_{\mathcal{G}}^{\Gamma}$ near $\phi_{x}$ as given by Lemma 2.2, Similarly, pick a linear orbifold chart $\left\{W_{\Phi_{0}(x)},(\mathcal{H})_{\Phi_{0}(x)}, \varpi_{\Phi_{0}(x)}\right\}$ 
for $|\mathcal{H}|$ at $\Phi_{0}(x)$; by shrinking charts if necessary, we may assume that $\Phi_{0}\left(V_{x}\right) \subseteq$ $W_{\Phi_{0}(x)}$. Then $\left\{W_{\Phi_{0}(x)}^{\left\langle\Phi_{* 0}\left(\phi_{x}\right)\right\rangle}, \kappa_{\Phi_{* 0}\left(\phi_{x}\right)}\right\}$ is a manifold chart for $\mathcal{S}_{\mathcal{H}}^{\Gamma}$ near $\Phi_{* 0}\left(\phi_{x}\right)$. As $\Phi_{1}$ commutes with each of the structure maps of $\mathcal{G}$ and $\mathcal{H}$, for each $y \in V_{x}^{\left\langle\phi_{x}\right\rangle}$ we have

$$
\begin{aligned}
\kappa_{\Phi_{* 0}\left(\phi_{x}\right)}^{-1} \circ \Phi_{* 0} \circ \kappa_{\phi_{x}}(y) & =\kappa_{\Phi_{* 0}\left(\phi_{x}\right)}^{-1} \circ \Phi_{1} \circ\left(\xi_{x}^{y}\right)^{-1} \circ \phi_{x} \\
& =\kappa_{\Phi_{* 0}\left(\phi_{x}\right)}^{-1} \circ\left(\left(\xi_{\mathcal{H}}\right)_{\Phi_{0}(x)}^{\Phi_{0}(y)}\right)^{-1} \circ \Phi_{1} \circ \phi_{x} \\
& =\kappa_{\Phi_{* 0}\left(\phi_{x}\right)}^{-1} \circ\left(\left(\xi_{\mathcal{H}}\right)_{\Phi_{0}(x)}^{\Phi_{0}(y)}\right)^{-1} \circ \Phi_{* 0}\left(\phi_{x}\right) \\
& =\Phi_{0}(y) .
\end{aligned}
$$

It follows that the map

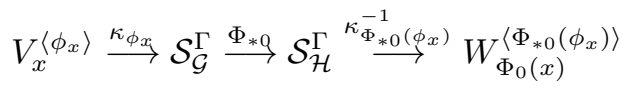

is nothing more than the restriction of $\Phi_{0}$ to $V_{x}^{\left\langle\phi_{x}\right\rangle} \subseteq G_{0}$, and hence is smooth. As this is true for each chart at each $\phi_{x} \in \mathcal{S}_{\mathcal{G}}^{\Gamma}, \Phi_{* 0}$ is a smooth map.

For each $\left(g, \phi_{x}\right) \in\left(\mathcal{G}^{\Gamma}\right)_{1}$, we set

$$
\Phi_{* 1}\left[\left(g, \phi_{x}\right)\right]=\left(\Phi_{1}(g), \Phi_{*}\left(\phi_{x}\right)\right) .
$$

In other words, $\Phi_{* 1}\left[\left(g, \phi_{x}\right)\right]$ is the arrow in $\left(\mathcal{H}^{\Gamma}\right)_{1}$ given by the action of $\Phi_{1}(g)$ on $\Phi_{*}\left(\phi_{x}\right)$. Then

$$
\begin{gathered}
s_{\mathcal{H}^{\Gamma}}\left(\Phi_{* 1}\left[\left(g, \phi_{x}\right)\right]\right)=\Phi_{*}\left(\phi_{x}\right)=\Phi_{* 0}\left(s_{\mathcal{G}^{\Gamma}}\left[\left(g, \phi_{x}\right)\right]\right), \\
t_{\mathcal{H}^{\Gamma}}\left(\Phi_{* 1}\left[\left(g, \phi_{x}\right)\right]\right)=\Phi_{*}\left(g \phi_{x}\right)=\Phi_{* 0}\left(t_{\mathcal{G}^{\Gamma}}\left[\left(g, \phi_{x}\right)\right]\right),
\end{gathered}
$$

etc., so that $\Phi_{* 0}$ and $\Phi_{* 1}$ commute with the structure maps $\mathcal{G}^{\Gamma}$ and $\mathcal{H}^{\Gamma}$. For each $\left(g, \phi_{x}\right) \in\left(\mathcal{G}^{\Gamma}\right)_{1}$, as $s_{\mathcal{G}^{\Gamma}}$ is a local diffeomorphism, there is a neighborhood $W_{g}$ of $g$ in $\left(\mathcal{G}^{\Gamma}\right)_{1}$ which is diffeomorphic to $V_{x}^{\left\langle\phi_{x}\right\rangle}$. Via this diffeomorphism and the corresponding construction for $\left(\mathcal{H}^{\Gamma}\right)_{1}$, just as in the case of $\Phi_{* 0}, \Phi_{* 1}$ corresponds to the restriction of $\Phi_{1}$ to the submanifold $W_{g}$ of $G_{1}$. It follows that $\Phi_{* 1}$ is smooth, and so $\Phi_{*}$ is a homomorphism of Lie groupoids.

Now, assume $\Phi$ is a strong equivalence. Then $\Phi_{0}: G_{0} \rightarrow H_{0}$ is a surjective submersion. Moreover, $\Phi_{1}$ restricts to an isomorphism from $G_{x}$ to $(\mathcal{H})_{\Phi_{0}(x)}$ for each $x \in G_{0}$. It follows that for each $x \in G_{0}$, the map $\Phi_{* 0}$ defined in equation (2.2) is a bijection between $\operatorname{HOM}\left(\Gamma, G_{x}\right)$ and $\operatorname{HOM}\left(\Gamma,(\mathcal{H})_{\Phi_{0}(x)}\right)$, so $\Phi_{* 0}$ is surjective. Within local charts, $\Phi_{* 0}$ is the restriction of a surjective submersion; hence, $\Phi_{* 0}$ is a surjective submersion. Moreover, the identification of $\mathcal{G}$ with the pullback of $\mathcal{H}$ via $\Phi_{0} \times \Phi_{0}: G_{0} \times G_{0} \rightarrow H_{0} \times H_{0}$ identifies the $\mathcal{G}$-action on $\mathcal{S}_{\mathcal{G}}^{\Gamma}$ with the pullback of the $\mathcal{H}$-action on $\mathcal{S}_{\mathcal{H}}^{\Gamma}$ via $\Phi_{* 0} \times \Phi_{* 0}: \mathcal{S}_{\mathcal{G}}^{\Gamma} \times \mathcal{S}_{\mathcal{G}}^{\Gamma} \rightarrow \mathcal{S}_{\mathcal{H}}^{\Gamma} \times \mathcal{S}_{\mathcal{H}}^{\Gamma}$. Hence, $\Phi_{*}$ is a strong equivalence.

Noting that the Morita equivalence class of an orbifold groupoid is the same as the Morita equivalence class via strong equivalences (see [1, page 21]), it follows that the orbifold structure of $\tilde{Q}_{\Gamma}$ depends only on $\Gamma$ and the orbifold structure of $Q$, but not on the choice of $\mathcal{G}$.

2.2. Connected components of the $\Gamma$-sectors. We now parameterize the space of $\Gamma$-sectors following [1, page 83]. 
Definition 2.6 (Equivalence in $\mathcal{G}^{\Gamma}$ ). Let $Q$ be an orbifold and $\Gamma$ a finitely generated group. Let $\phi_{x}, \psi_{y} \in \mathcal{S}_{\mathcal{G}}^{\Gamma}$ and suppose there is a linear chart $\left\{V_{x}, G_{x}, \pi_{x}\right\}$ at $x$ for $Q$ with $y \in V_{x}$. We say that $\phi_{x}$ locally covers $\psi_{y}$ with respect to the linear chart $\left\{V_{x}, G_{x}, \pi_{x}\right\}$, written $\phi_{x} \stackrel{\text { loc }}{\curvearrowright} \psi_{y}$, if there is a $g \in G_{x}$ such that $g\left[\left(\xi_{x}^{y} \circ \psi_{y}\right)(\gamma)\right] g^{-1}=$ $\phi_{x}(\gamma)$ for each $\gamma \in \Gamma$. When we say that $\phi_{x}$ locally covers $\psi_{y}$, we mean that there exists a linear chart with respect to which $\phi_{x}$ locally covers $\psi_{y}$.

Extending this to an equivalence relation on all of $\mathcal{G}^{\Gamma}$, we say that two $\mathcal{G}$-orbits of homomorphisms $\mathcal{G} \phi_{x}$ and $\mathcal{G} \psi_{y}$ are equivalent, written $\mathcal{G} \phi_{x} \approx \mathcal{G} \psi_{y}$, if there is a finite sequence $\phi_{x_{0}}, \phi_{x_{1}}, \ldots, \phi_{x_{l}}$ such that $\phi_{x_{0}} \in \mathcal{G} \phi_{x}, \phi_{x_{l}} \in \mathcal{G} \psi_{y}$, and for each $i$, $\phi_{x_{i}} \stackrel{\text { loc }}{\curvearrowright} \phi_{x_{i+1}}$ or $\phi_{x_{i+1}} \stackrel{\text { loc }}{\curvearrowright} \phi_{x_{i}}$. We let $\left(\phi_{x}\right)$ denote the $\approx$-class of $\mathcal{G} \phi_{x}$; we will refer to this class simply as $(\phi)$ when there is no specific representative $\phi_{x}$ in mind or to emphasize the lack of dependence on an $x \in G_{0}$. We let $T_{Q}^{\Gamma}$ denote the set of $\approx$-classes in $\mathcal{S}_{\mathcal{G}}^{\Gamma}$.

Note that two homomorphisms are equivalent only if they are connected by a sequence of local coverings in linear orbifold charts. Allowing charts of the form $M / G$ where $M$ is a manifold and $G$ a finite group results in a different definition.

The following two lemmas allow us to simplify the definition of $\approx$ when dealing with $\mathcal{G}$-orbits of elements of $\mathcal{S}_{\mathcal{G}}^{\Gamma}$ rather than points in $\mathcal{S}_{\mathcal{G}}^{\Gamma}$ themselves. Lemma 2.7 shows that by picking an appropriate representative of an orbit, the conjugation in the definition of $\stackrel{\text { loc }}{\curvearrowright}$ is unnecessary. Lemma 2.8 shows that the definition of $\stackrel{\text { loc }}{\curvearrowright}$ is as well-defined on orbits as it can be; i.e. it holds for all elements of an orbit that have representatives in the same linear chart.

Lemma 2.7. Let $Q$ be an orbifold and $\Gamma$ a finitely generated group, and take $\phi_{x}, \psi_{y} \in \mathcal{S}_{\mathcal{G}}^{\Gamma}$. If $\phi_{x} \stackrel{\text { loc }}{\curvearrowright} \psi_{y}$ with respect to the chart $\left\{V_{x}, G_{x}, \pi_{x}\right\}$, then there is an element $\psi_{y^{\prime}}$ of the $\mathcal{G}$-orbit of $\psi_{y}$ such that $y^{\prime} \in V_{x}$ and

$$
\xi_{x}^{y^{\prime}} \circ \psi_{y^{\prime}}=\phi_{x} .
$$

Proof. Suppose there is a linear chart and a $g \in G_{x}$ such that $g\left[\left(\xi_{x}^{y} \circ \psi_{y}\right)(\gamma)\right] g^{-1}=$ $\phi_{x}(\gamma)$ for each $\gamma \in \Gamma$. As $\xi_{x}$ defines a surjective map from $s^{-1}(y) \cap t^{-1}\left(V_{x}\right)$ onto $G_{x}$, there is an arrow $h \in G_{1}$ such that $s(h)=y, t(h) \in V_{x}$, and $\xi_{x}(h)=g$. Therefore, for each $\gamma \in \Gamma$, recalling that $\xi_{x}^{y}$ is simply the restriction of $\xi_{x}$ to $G_{y}$,

$$
\begin{aligned}
\phi_{x}(\gamma)= & g\left[\left(\xi_{x}^{y} \circ \psi_{y}\right)(\gamma)\right] g^{-1} \\
= & \xi_{x}(h)\left[\left(\xi_{x}^{y} \circ \psi_{y}\right)(\gamma)\right] \xi_{x}\left(h^{-1}\right) \\
= & \xi_{x}\left[h\left(\psi_{y}(\gamma)\right) h^{-1}\right] \\
= & \xi_{x}^{t(h)}\left[h\left(\psi_{y}(\gamma)\right) h^{-1}\right] \\
& \left(\operatorname{as} h\left(\psi_{y}(\gamma)\right) h^{-1} \in G_{t(h)}\right) \\
= & {\left[\xi_{x}^{t(h)}\left(h \psi_{y}\right)\right](\gamma) . }
\end{aligned}
$$

Setting $y^{\prime}=t(h)$ and $\psi_{y^{\prime}}=h \psi_{y}$, we are done.

Lemma 2.8. Let $Q$ be an orbifold and $\Gamma$ a finitely generated group, and take $\phi_{x}, \psi_{y} \in \mathcal{S}_{\mathcal{G}}^{\Gamma}$. If $\phi_{x} \stackrel{\text { loc }}{\curvearrowright} \psi_{y}$, then $\phi_{x}$ locally covers every element of the $\mathcal{G}$-orbit of $\psi_{y}$ in $\beta_{\Gamma}^{-1}\left(V_{x}\right)$.

Proof. Suppose $\phi_{x} \stackrel{\text { loc }}{\curvearrowright} \psi_{y}$ so that there is a $g \in G_{x}$ such that for each $\gamma \in \Gamma$,

$$
\phi_{x}(\gamma)=g\left[\left(\xi_{x}^{y} \circ \psi_{y}\right)(\gamma)\right] g^{-1} .
$$


Each element of the $\mathcal{G}$-orbit of $\psi_{y}$ in $\beta_{\Gamma}^{-1}\left(V_{x}\right)$ is of the form $h \psi_{y}$ for some $h \in G_{1}$ with $s(h)=y$ and $t(h) \in V_{x}$. Fixing one such $h \psi_{y}$, we have for each $\gamma \in \Gamma$ that

$$
\begin{aligned}
g \xi_{x}\left(h^{-1}\right)\left[\left(\xi_{x}^{t(h)} \circ\left(h \psi_{y}\right)\right)(\gamma)\right]\left[g \xi_{x}\left(h^{-1}\right)\right]^{-1} \\
=g \xi_{x}\left(h^{-1}\right)\left[\xi_{x}^{t(h)}\left(h \psi_{y}(\gamma) h^{-1}\right)\right] \xi_{x}(h) g^{-1} \\
=g \xi_{x}\left(h^{-1} h\right)\left[\left(\xi_{x}^{y} \circ \psi_{y}\right)(\gamma)\right] \xi_{x}\left(h^{-1} h\right) g^{-1} \\
=g\left[\left(\xi_{x}^{y} \circ \psi_{y}\right)(\gamma)\right] g^{-1} \\
=\phi_{x}(\gamma) .
\end{aligned}
$$

As $g \xi_{x}\left(h^{-1}\right) \in G_{x}$, it follows that $\phi_{x} \stackrel{l o c}{\curvearrowright} h \psi_{y}$.

Lemma 2.9. Suppose $Q$ is a compact orbifold and $\Gamma$ is a finitely generated group. Then $T_{Q}^{\Gamma}$ is finite.

Proof. For each $p \in Q$, pick a linear chart $\left\{V_{x}, G_{x}, \pi_{x}\right\}$ at $p$. Then the collection $\left\{U_{p}: p \in Q\right\}$ forms an open cover of $Q$. As $Q$ is compact, pick a finite subcover corresponding to the points $x_{1}, x_{2}, \ldots x_{k} \in G_{0}$ with respective orbits $\sigma\left(x_{i}\right)=p_{i} \in$ $Q$. We claim that each $(\psi) \in T_{Q}^{\Gamma}$ has a representative in the set

$$
\mathcal{H}=\bigcup_{i=1}^{k} \operatorname{HOM}\left(\Gamma, G_{x_{i}}\right),
$$

which is clearly finite.

Let $\psi_{y}: \Gamma \rightarrow G_{y}$ be an arbitrary element of $\mathcal{S}_{\mathcal{G}}^{\Gamma}$ and let $q=\sigma(y) \in Q$ denote the orbit of $y$. Then there is an $i$ such that $q \in U_{p_{i}}$; hence, there is an $h \in G_{1}$ with $s(h)=y$ and $t(h) \in V_{x_{i}}$. Recalling that $\xi_{x}^{t(h)}: G_{t(h)} \rightarrow G_{x}$ is an injective group homomorphism, define $\phi_{x}=\xi_{x}^{t(h)} \circ\left(h \psi_{y}\right)$, i.e.

$$
\phi_{x}: \Gamma \stackrel{h \psi_{y}}{\longrightarrow} G_{t(h)} \stackrel{\xi_{x}^{t(h)}}{\longrightarrow} G_{x}
$$

and then $\phi_{x}$ is an element of $\mathcal{H}$.

That $\phi_{x} \stackrel{\text { loc }}{\curvearrowright} h \psi_{y}$ is obvious from the definition of $\phi_{x}$. Hence, as $h \psi_{y} \in \mathcal{G} \psi_{y}$, this implies that $\phi_{x} \approx \psi_{y}$.

If $\phi_{x} \stackrel{l o c}{\curvearrowright} \phi_{y}$ with respect to the linear chart $\left\{V_{x}, G_{x}, \pi_{x}\right\}$, then by Lemma 2.7, the $G_{x}$-orbit of $y$ in $V_{x}$ intersects $V_{x}^{\left\langle\phi_{x}\right\rangle}$. As $G_{x}$ acts linearly so that $V_{x}^{\left\langle\phi_{x}\right\rangle}$ is a subspace, it follows that $\phi_{x}$ and $\phi_{y}$ represent $\mathcal{G}$-orbits in the same connected component of $\tilde{Q}_{\Gamma}$. If $\phi_{x} \approx \phi_{y}$, then they are connected by a finite sequence of points related by local coverings. For each $i$, the two points $C_{G_{x_{i}}}\left(\phi_{x_{i}}\right) x_{i}$ and $C_{G_{x_{i+1}}}\left(\phi_{x_{i+1}}\right) x_{i+1}$ lie in the same connected component of $\tilde{Q}_{\Gamma}$ regardless of the direction of the covering, so the $\mathcal{G}$-orbits of $\phi_{x}$ and $\phi_{y}$ lie in the same connected component of $\tilde{Q}_{\Gamma}$.

Conversely, we take each chart for $\tilde{Q}_{\Gamma}$ to be of the form $\left\{V_{x}^{\left\langle\phi_{x}\right\rangle}, C_{G_{x}}\left(\phi_{x}\right), \pi_{x}^{\phi_{x}}\right\}$, induced from a chart $\left\{V_{x}, G_{x}, \pi_{x}\right\}$ at $x$; then the image of the injective homomorphism $\xi_{x}^{y}: G_{y} \rightarrow G_{x}$ contains $\operatorname{Im} \phi_{x}$ if and only if $y \in V_{x}^{\left\langle\phi_{x}\right\rangle}$. Hence, this chart defines a local covering by $\phi_{x}$ of each homomorphism $\phi_{y}=\left(\xi_{x}^{y}\right)^{-1} \circ \phi_{x}$ corresponding to a $y \in V_{x}^{\left\langle\phi_{x}\right\rangle}$. If $\phi_{x}$ and $\phi_{y}$ represent points whose orbits are in the same connected component of $\tilde{Q}_{\Gamma}$, then there is a path connecting the orbits of $\phi_{x}$ and 
$\phi_{y}$. Pick a linear chart for each point on the path and then a finite subcover of these uniformized sets. It follows that there is a finite sequence of local equivalences connecting $\phi_{x}$ to $\phi_{y}$. With this, we make the following definition.

Definition 2.10 ( $\Gamma$-sector). Let $\tilde{Q}_{(\phi)}$ denote the subset of $\tilde{Q}_{\Gamma}$ corresponding to orbits of points in the $\approx$-class $(\phi)$. Then

$$
\tilde{Q}_{\Gamma}=\bigsqcup_{(\phi) \in T_{Q}^{\Gamma}} \tilde{Q}_{(\phi)}
$$

is a decomposition of $\tilde{Q}_{\Gamma}$ into connected components. We call $\tilde{Q}_{(\phi)}$ the $\Gamma$-sector corresponding to $(\phi)$.

We let $\pi: \tilde{Q}_{\Gamma} \rightarrow Q$ denote the map $\pi\left(\mathcal{G} \phi_{x}\right)=\sigma(x)$ that sends the orbit $\mathcal{G} \phi_{x}$ in $\tilde{Q}_{\Gamma}$ to the orbit of $x$ in $Q$. Note that $\pi$ is the map on orbit spaces induced by $\beta_{\Gamma}$ and hence is a smooth map of orbifolds.

2.3. Euler-Satake characteristics and the $\Gamma$-Euler-Satake class. If the orbifold $Q$ is closed, we let $\chi_{E S}(Q)$ denote the Euler-Satake characteristic of $Q$ (see [15] where this number is called the Euler characteristic of $Q$ as a $V$-manifold or [16] where this quantity is denoted by $\left.\chi_{\text {orb }}(Q)\right)$. Recall that the Euler-Satake characteristic is most easily defined in terms of a simplicial decomposition $\mathcal{T}$ of $Q$ such that the isomorphism class of the isotropy group is constant on the interior of each simplex (it is shown in [10 that such a simplicial decomposition always exists). For each simplex $\sigma \in \mathcal{T}$, let $G_{\sigma}$ denote the (isomorphism class of the) isotropy group on the interior of $\sigma$. Then the Euler-Satake characteristic of $Q$ is the rational number

$$
\chi_{E S}(Q)=\sum_{\sigma \in \mathcal{T}}(-1)^{\operatorname{dim} \sigma} \frac{1}{\left|G_{\sigma}\right|} .
$$

Note that this differs from $\chi_{t o p}(Q)$, the ordinary Euler characteristic of the underlying topological space $\mathbb{X}_{Q}$ of $Q$, in that

$$
\chi_{\text {top }}(Q)=\sum_{\sigma \in \mathcal{T}}(-1)^{\operatorname{dim} \sigma} .
$$

Recall (see [1, Definition 2.25 and Definition 2.28, pages 44-45]; see also [3]) that an orbifold vector bundle over $Q$ of rank $k$ is given by a $\mathcal{G}$-vector bundle $\rho: E \rightarrow G_{0}$ of rank $k$ such that each $g \in G_{1}$ induces a linear isomorphism of fibers $g: \rho^{-1}(s(g)) \rightarrow \rho^{-1}(t(g))$. Sections of this orbibundle correspond to $\mathcal{G}$-invariant sections $\omega: G_{0} \rightarrow E$. We denote the translation groupoid by $\mathcal{E}=\mathcal{G} \ltimes E$. A bundle $E$ is called good if for each $x \in G_{0}, \operatorname{Ker}\left(G_{x}\right)$ acts trivially on each fiber $E_{x}$, where $\operatorname{Ker}\left(G_{x}\right)$ denotes the set of constant arrows in $G_{x}$, i.e. the arrows $g \in G_{x}$ such that there is a neighborhood of $g$ in $G_{1}$ on which $s=t$. Within a linear chart over which $E$ is trivial, this means that the kernel of the group action on the base space coincides with the kernel of the group action on the total space.

Note that as $\mathcal{E}$ is an orbifold structure for $|\mathcal{E}|$, we can apply the construction of $\Gamma$-sectors to form $|\widetilde{\mathcal{E}}|_{\Gamma}=\left|\mathcal{E}^{\Gamma}\right|$ where $\mathcal{E}^{\Gamma}=\mathcal{E} \ltimes \mathcal{S}_{\mathcal{E}}^{\Gamma}$.

\footnotetext{
${ }^{1}$ We avoid using this notation here, as it is more frequently used in the literature to denote the stringy orbifold Euler characteristic of $Q$.
} 
Lemma 2.11. Let $\rho: E \rightarrow G_{0}$ be an orbifold vector bundle of rank $k$, and let $\Gamma$ be a finitely generated group. Then $\mathcal{S}_{\mathcal{E}}^{\Gamma}$ is naturally a $\mathcal{G}^{\Gamma}$-vector bundle over $\mathcal{S}_{\mathcal{G}}^{\Gamma}$ making $\left.\widetilde{\mid \mathcal{E}}\right|_{\Gamma}$ into an orbifold vector bundle over $\tilde{Q}_{\Gamma}$. An orientation of $E$ induces an orientation of $\mathcal{S}_{\mathcal{E}}^{\Gamma} . A \mathcal{G}$-invariant section $\omega: G_{0} \rightarrow E$ naturally induces an $\mathcal{E}^{\Gamma}$-invariant section $\tilde{\omega}_{\Gamma}: \mathcal{S}_{\mathcal{G}}^{\Gamma} \rightarrow \mathcal{S}_{\mathcal{E}}^{\Gamma}$. Within corresponding linear charts for $\mathcal{E}^{\Gamma}$ and $\mathcal{E}, \tilde{\omega}_{\Gamma}$ is simply the restriction of $\omega$ to a subspace; in particular $\tilde{\omega}_{\Gamma}\left(\phi_{x}\right)=0$ if and only if $\omega(x)=0$.

Proof. As in the proof of Lemma 2.5, we let $(\mathcal{E})_{1}$ denote the space of arrows $\mathcal{E},(\mathcal{E})_{e}$ the isotropy group of $e \in E$, and $s_{\mathcal{E}}$ and $t_{\mathcal{E}}$ the source and target maps, respectively. Note that $E$ is the space of objects of $\mathcal{E}$. An element of $(\mathcal{E})_{1}$ is given by a $g \in G_{1}$ and an $e \in \rho^{-1}(s(g)) \subseteq E$; we will denote this arrow by $(g, e)$. For each $e \in E$, the map

$$
\begin{aligned}
& \rho_{1}:(\mathcal{E})_{1} \longrightarrow G_{1} \\
& :(g, e) \longmapsto g
\end{aligned}
$$

restricts to an injective homomorphism from the isotropy group $(\mathcal{E})_{e}$ into the isotropy group $G_{\rho(e)}$ of $\rho(e) \in G_{0}$. Regarding the space of $\Gamma$-sectors, the space of objects in $\mathcal{E}^{\Gamma}$ is $\mathcal{S}_{\mathcal{E}}^{\Gamma}$. We show that $\mathcal{S}_{\mathcal{E}}^{\Gamma}$ is a $\mathcal{G}^{\Gamma}$-vector bundle over $\mathcal{S}_{\mathcal{G}}^{\Gamma}$ with the desired properties.

Each element of $\mathcal{S}_{\mathcal{E}}^{\Gamma}$ is a homomorphism $\phi_{e}: \Gamma \rightarrow(\mathcal{E})_{e}$ where $e \in E$. We define

$$
\begin{aligned}
\tilde{\rho}_{\Gamma}: \mathcal{S}_{\mathcal{E}}^{\Gamma} & \longrightarrow \mathcal{S}_{\mathcal{G}}^{\Gamma} \\
: \phi_{e} & \longmapsto \rho_{1} \circ \phi_{e} .
\end{aligned}
$$

Then $\tilde{\rho}_{\Gamma}\left(\phi_{e}\right): \Gamma \rightarrow G_{\rho(e)}$ is a homomorphism for each $\phi_{e} \in \mathcal{S}_{\mathcal{E}}^{\Gamma}$.

A linear chart for $\left|\mathcal{E}^{\Gamma}\right|$ whose image contains $\phi_{e}$ is given as follows. Let $x=$ $\rho(e) \in G_{0}$. Then there is a linear chart $\left\{V_{x}, G_{x}, \pi_{x}\right\}$ for $Q$ at $x$. By shrinking $V_{x}$ if necessary, we may assume that $\left.E\right|_{V_{x}}$ is trivial as a vector bundle over $V_{x}$ (although the $G_{x}$-structure need not be trivial). Then $\left\{V_{x} \times \mathbb{R}^{k}, G_{x}, \tilde{\pi}_{x}\right\}$ is a linear chart for $|\mathcal{E}|$ whose image contains $e$, with $\tilde{\pi}_{x}\left(V_{x} \times \mathbb{R}^{k}\right)=\rho^{-1}\left(V_{x}\right) \subseteq E$. If $\operatorname{proj}_{V_{x}}: V_{x} \times \mathbb{R}^{k} \rightarrow V_{x}$ denotes the projection onto the first factor, then $\pi_{x} \circ \operatorname{proj}_{V_{x}}=\rho \circ \tilde{\pi}_{x}$.

Note that we are not interested in linear charts for $\mathcal{E}$ at $e$, as such charts do not respect the structure of $E$ as a rank $k$ vector bundle over $G_{0}$. Specifically, if $e$ does not correspond to an element of the zero-section in $E$, then a chart in which $e$ corresponds to the identity in $\mathbb{R}^{n} \times \mathbb{R}^{k}$ would not give a local trivialization of $E$. Rather, $\left\{V_{x} \times \mathbb{R}^{k}, G_{x}, \tilde{\pi}_{x}\right\}$ is a linear chart whose image contains complete fibers of $E$ such that $V_{x} \times\{0\}$ corresponds to the zero-section and the origin in $V_{x} \times \mathbb{R}^{n}$ corresponds to $x$ through the injection of $G_{0}$ into $E$ as the zero-section.

By Lemma 2.4, a chart for $|\widetilde{\mathcal{E}}|_{\Gamma}=\left|\mathcal{E}^{\Gamma}\right|$ whose image contains $\phi_{e}$ is of the form $\left\{\left(V_{x} \times \mathbb{R}^{k}\right)^{\left\langle\phi_{e}\right\rangle}, C_{G_{x}}\left(\phi_{e}\right), \tilde{\pi}_{x}^{\phi_{e}}\right\}$, and a linear chart for $\tilde{Q}_{\Gamma}=\left|\mathcal{G}^{\Gamma}\right|$ at $\tilde{\rho}_{\Gamma}\left(\phi_{e}\right)$ is of the form $\left\{V_{x}^{\left\langle\tilde{\rho}_{\Gamma}\left(\phi_{e}\right)\right\rangle}, C_{G_{x}}\left(\tilde{\rho}_{\Gamma}\left(\phi_{e}\right)\right), \pi_{x}^{\tilde{\rho}_{\Gamma}\left(\phi_{e}\right)}\right\}$. Since $G_{x}$ acts linearly on each fiber of $V_{x} \times \mathbb{R}^{k}$ and $G_{x}\left(V_{x} \times\{0\}\right)=\left(V_{x} \times\{0\}\right)$, we have

$$
\begin{aligned}
\left(V_{x} \times \mathbb{R}^{k}\right)^{\left\langle\phi_{e}\right\rangle} & =\left(V_{x} \times\{0\}\right)^{\left\langle\phi_{e}\right\rangle} \times\left(\mathbb{R}^{k}\right)^{\left\langle\phi_{e}\right\rangle} \\
& =V_{x}^{\left\langle\tilde{\rho}_{\Gamma}\left(\phi_{e}\right)\right\rangle} \times\left(\mathbb{R}^{k}\right)^{\left\langle\phi_{e}\right\rangle} .
\end{aligned}
$$

By construction, $\tilde{\pi}_{x}^{\tilde{\rho}_{\Gamma}\left(\phi_{e}\right)} \circ \operatorname{proj}_{V_{x}^{\left\langle\phi_{e}\right\rangle}}=\tilde{\rho}_{\Gamma} \circ \tilde{\pi}_{x}^{\phi_{e}}$. As such charts exist at each point $\phi_{e} \in \mathcal{S}_{\mathcal{E}}^{\Gamma}$ and as they clearly transform appropriately, being restrictions of charts 
for $E, \mathcal{S}_{\mathcal{E}}^{\Gamma}$ is a $\mathcal{G}^{\Gamma}$-vector bundle over $\mathcal{S}_{\mathcal{G}}^{\Gamma}$. Moreover, an orientation of $E$ obviously induces an orientation of $\mathcal{S}_{\mathcal{E}}^{\Gamma}$ by restriction within charts.

Now, let $\left(g, \phi_{x}\right) \in\left(\mathcal{G}^{\Gamma}\right)_{1}$ where $g \in G_{0}$ with $s(g)=x$. Note that $s_{\mathcal{G}^{\Gamma}}\left[\left(g, \phi_{x}\right)\right]=\phi_{x}$ is a homomorphism $\Gamma \rightarrow G_{x}$. Hence, $\left(g, \phi_{x}\right)$ induces a map

$$
\left(g, \phi_{x}\right): \tilde{\rho}_{\Gamma}^{-1}\left(\phi_{x}\right) \longrightarrow \tilde{\rho}_{\Gamma}^{-1}\left(t\left(\phi_{x}\right)\right) .
$$

With respect to a chart for $\mathcal{E}^{\Gamma}$ as above, $\tilde{\rho}_{\Gamma}^{-1}\left(\phi_{x}\right)=\left(\rho^{-1}(x)\right)^{\left\langle\phi_{x}\right\rangle}$ and $\tilde{\rho}_{\Gamma}^{-1}\left(t\left(\phi_{x}\right)\right)=$ $\left(\rho^{-1}[t(g)]\right)^{\left\langle g \phi_{x}\right\rangle}$, so $\left(g, \phi_{x}\right)$ is simply the restriction of the linear isomorphism $g: \rho^{-1}(s(g)) \rightarrow \rho^{-1}(t(g))$ onto the invariant subspace $\left(\rho^{-1}(x)\right)^{\left\langle\phi_{x}\right\rangle}$ with image $\left(\rho^{-1}[t(g)]\right)^{\left\langle g \phi_{x}\right\rangle}$. Therefore, it is a linear isomorphism, and $\tilde{\rho}_{\Gamma}: \mathcal{S}_{\mathcal{E}}^{\Gamma} \rightarrow \mathcal{S}_{\mathcal{G}}^{\Gamma}$ defines an orbifold vector bundle $\left.\widetilde{\mid \mathcal{E}}\right|_{\Gamma} \rightarrow \tilde{Q}_{\Gamma}$.

A section $Q \rightarrow|\mathcal{E}|$ is a $\mathcal{G}$-invariant section $\omega: G_{0} \rightarrow E$. For each $x \in G_{0}$, as $\omega(x)$ is $G_{x}$-invariant, the map $\rho_{1}$ defined in equation (2.3) maps the isotropy group $(\mathcal{E})_{\omega(x)}$ of $\omega(x)$ isomorphically onto $G_{x}$. Given a section $\omega$, we define

$$
\tilde{\omega}_{\Gamma}: \mathcal{S}_{\mathcal{G}}^{\Gamma} \longrightarrow \mathcal{S}_{\mathcal{E}}^{\Gamma}
$$

by $\left[\tilde{\omega}_{\Gamma}\left(\phi_{x}\right)\right](\gamma)=\left(\left.\rho_{1}\right|_{(\mathcal{E})_{\omega(x)}}\right)^{-1}\left[\phi_{x}(\gamma)\right]$ for each $\gamma \in \Gamma$. In a chart for $\mathcal{E}^{\Gamma}$ of the form $\left\{\left(V_{x} \times \mathbb{R}^{k}\right)^{\left\langle\tilde{\omega}_{\Gamma}\left(\phi_{x}\right)\right\rangle}, C_{(\mathcal{E})_{\omega(x)}}\left(\tilde{\omega}_{\Gamma}\left(\phi_{x}\right)\right), \tilde{\pi}_{x}^{\tilde{\omega}_{\Gamma}\left(\phi_{x}\right)}\right\}$, the value of $\tilde{\omega}_{\Gamma}(y)$ coincides with that of $\omega(y)$ so that $\tilde{\omega}_{\Gamma}$ is, within a chart, just the restriction of $\omega$ to the invariant subspace $\left(V_{x} \times\{0\}\right)^{\left\langle\tilde{\omega}_{\Gamma}\left(\phi_{x}\right)\right\rangle}=V_{x}^{\left\langle\phi_{x}\right\rangle}$. Hence $\tilde{\omega}_{\Gamma}$ is a $\mathcal{G}^{\Gamma}$-invariant section of $\mathcal{S}_{\mathcal{E}}^{\Gamma}$. Moreover, as it is locally just a restriction, $\tilde{\omega}_{\Gamma}\left(\phi_{x}\right)=0$ if and only if $\omega(x)=0$.

Note that the bundle $\tilde{\rho}_{\Gamma}: \mathcal{S}_{\mathcal{E}}^{\Gamma} \rightarrow \mathcal{S}_{\mathcal{G}}^{\Gamma}$ is not simply the pullback of the bundle $E$ via $\beta_{\Gamma}$; it generally has different ranks over different connected components. It is easy to see that the operations of forming the tangent bundle, cotangent bundle, and exterior powers commute with the operation of forming the $\Gamma$-sectors; that is,

$$
\begin{aligned}
T\left(\tilde{Q}_{\Gamma}\right) & =\widetilde{(T Q)_{\Gamma}}, \\
T^{*}\left(\tilde{Q}_{\Gamma}\right) & \left.=\widetilde{\left(T^{*} Q\right.}\right)_{\Gamma},
\end{aligned}
$$

etc. These are, however, all good vector bundles. We note that it is possible that the orbifold vector bundle given by $\rho: E \rightarrow G_{0}$ is a good vector bundle while the induced bundle on $\Gamma$-sectors is not. We illustrate this with the following example.

Example 2.12. Let $G_{0}=\mathbb{C}$ and $F=\mathbb{C}^{2}$ with basis $\left\{f_{1}, f_{2}\right\}$ (note that we only use the complex structure to simplify notation). Let $E=G_{0} \times F$ be the trivial bundle and $\rho: E \rightarrow G_{0}$ the projection. Let $G=\mathbb{Z}_{2} \oplus \mathbb{Z}_{3}=\left\langle\alpha_{1}\right\rangle \oplus\left\langle\alpha_{2}\right\rangle$ act as follows. On $G_{0}, \alpha_{1}$ acts as multiplication by -1 and $\alpha_{2}$ acts as multiplication by $e^{2 \pi i / 3}$. On $E$, we let $\alpha_{1}\left(y, c_{1} f_{1}+c_{2} f_{2}\right)=\left(\alpha_{1} y,-c_{1} f_{1}+c_{2} f_{2}\right)$ and $\alpha_{2}\left(y, c_{1} f_{1}+c_{2} f_{2}\right)=$ $\left(\alpha_{2} y, c_{1} f_{1}+e^{2 \pi i / 3} c_{2} f_{2}\right)$. Then $E$ defines an orbifold vector bundle over the orbifold $Q=G \ltimes G_{0}$. Note that the kernel of the action on both $G_{0}$ and $E$ is trivial, so that $E \rightarrow G_{0}$ is a good orbifold vector bundle.

Let $x$ denote the origin in $G_{0}$ and let $\Gamma=\mathbb{Z}$ be generated by $\gamma$. Define $\phi_{x}: \mathbb{Z} \rightarrow G$ by $\phi_{x}: \gamma \mapsto \alpha_{1}$ and let $(\phi)$ denote the $\approx$-class of $\phi_{x}$ as usual. Then a chart for $\tilde{Q}_{(\phi)}$ at the orbit of $\phi_{x}$ is simply the origin, with $\alpha_{1}$ and $\alpha_{2}$ acting trivially. The kernel of the action is all of $G$.

Let $e$ denote the origin in $\rho^{-1}(x)$, and define $\phi_{e}: \gamma \mapsto \alpha_{1}$. Then $\tilde{\rho}_{\Gamma}\left(\phi_{e}\right)=\phi_{x}$. A chart for the fiber of $\left.\widetilde{\mathcal{E}}\right|_{\Gamma}$ at the orbit of $\phi_{y}$ is $\operatorname{span}_{\mathbb{C}}\left(f_{2}\right)$ where $\alpha_{1}$ acts trivially and $\alpha_{2}$ acts by multiplication by $e^{2 \pi i / 3}$. The kernel of the action is $\left\langle\alpha_{1}\right\rangle$. 
We see that $E \rightarrow G_{0}$ is a good vector bundle, while the induced bundle over the space of $\mathbb{Z}$-sectors is a bad vector bundle; on the connected component $\tilde{Q}_{(\phi)}$, the kernel of the action on the fiber is a proper subgroup of the kernel of the action on the base space.

Definition 2.13 ( $\Gamma$-cohomology). Let $Q$ be an orbifold and $\Gamma$ a finitely generated group. We let $H_{\Gamma}^{*}(Q)$ denote the (singular or de Rham) cohomology of $\tilde{Q}_{\Gamma}$. If $Q$ admits an almost-complex structure, we let $H_{o r b, \Gamma}^{*}(Q)$ denote the Chen-Ruan cohomology of $\tilde{Q}_{\Gamma}$ (see [4]). Throughout, we use real coefficients.

Assume $Q$ is oriented, inducing an orientation of $\tilde{Q}_{\Gamma}$. Endow $\tilde{Q}_{\Gamma}$ with a Riemannian metric and metric connection $\tilde{\omega}$ with curvature $\tilde{\Omega}$. Let $E(\tilde{\Omega})$ denote the Euler curvature form (see [15] or [16]). Note that if (1) denotes the $\approx$-class of the trivial homomorphism into any isotropy group, then as all such homomorphisms are clearly elements of the same $\approx$-class, $\tilde{Q}_{(1)}$ is clearly diffeomorphic to $Q$. Restricting to the connected component $\tilde{Q}_{(1)}$, we have a metric connection $\omega$ on $Q$ with curvature $\Omega$ and Euler curvature form $E(\Omega)$.

Definition 2.14 ( $\Gamma$-Euler-Satake class). Let $Q$ be an oriented orbifold of dimension $n$ and $\Gamma$ a finitely generated group. Let $e_{E S}(Q) \in H^{n}(Q)$ denote the cohomology class of the Euler curvature form on $Q$. We refer to it as the Euler-Satake class of $Q$. Let $e_{\Gamma}^{E S}(Q) \in H_{\Gamma}^{*}(Q)$ denote the cohomology class of the Euler curvature form on $\tilde{Q}_{\Gamma}$, called the $\Gamma$-Euler-Satake class of $Q$.

Note that the Euler-Satake class and $\Gamma$-Euler-Satake class can be defined in the obvious analogous manner for any good, oriented orbifold vector bundle over $Q$. Moreover, for bad, oriented orbifold vector bundles, we can use the techniques in [18. We are using the usual convention that the $\Gamma$-Euler-Satake class of $Q$ indicates the $\Gamma$-Euler-Satake class of the tangent bundle of $Q$.

The class $e_{E S}(Q)$ is the cohomology class represented by the Gauss-Bonnet integrand in [15], while $e_{\mathbb{Z}}^{E S}(Q)$ is the cohomology class represented by the GaussBonnet integrand in [16, Theorem 3.2]. We have

$$
e_{\Gamma}^{E S}(Q)=\sum_{(\phi) \in T_{Q}^{\Gamma}} e_{E S}\left(\tilde{Q}_{(\phi)}\right)
$$

so that $e_{\Gamma}^{E S}(Q)$ is generally not a homogeneous cohomology class. Satake's GaussBonnet theorem for orbifolds implies that if $Q$ is compact, then

$$
\left\langle e_{E S}\left(\tilde{Q}_{(\phi)}\right) ;\left[\tilde{Q}_{(\phi)}\right]\right\rangle=\chi_{E S}\left(\tilde{Q}_{(\phi)}\right) .
$$

$\operatorname{By}\left\langle e_{E S}\left(\tilde{Q}_{(\phi)}\right) ;\left[\tilde{Q}_{(\phi)}\right]\right\rangle$ we mean the integral of any differential form representing $e_{E S}\left(\tilde{Q}_{(\phi)}\right)$ on $\tilde{Q}_{(\phi)}$. In particular, $e_{\Gamma}^{E S}(Q)=0$ implies that $\chi_{E S}\left(\tilde{Q}_{(\phi)}\right)=0$ for each $(\phi) \in T_{Q}^{\Gamma}$.

Conversely, since the top cohomology group of each $\Gamma$-sector is isomorphic to $\mathbb{R}$ in the oriented case (see [1, page 34 and Theorem 2.13]), $\chi_{E S}\left(\tilde{Q}_{(\phi)}\right)=0$ implies that $e_{E S}\left(\tilde{Q}_{(\phi)}\right)=0$. If this is true for each $(\phi) \in T_{Q}^{\Gamma}$, then by equation (2.4), $e_{\Gamma}^{E S}(Q)=0$. We summarize this observation as follows. 
Lemma 2.15. Let $Q$ be a closed, oriented orbifold and $\Gamma$ a finitely generated group. Then $e_{\Gamma}^{E S}(Q)=0$ if and only if $\chi_{E S}\left(\tilde{Q}_{(\phi)}\right)=0$ for each $(\phi) \in T_{Q}^{\Gamma}$.

\section{Topological Properties of the $\Gamma$-SeCtors}

As above, $Q$ is an $n$-dimensional orbifold whose orbifold structure is given by the groupoid $\mathcal{G}$. Recall from Subsection 2.2 that $\pi: \tilde{Q}_{\Gamma} \rightarrow Q$ is the smooth map $\pi\left(\mathcal{G} \phi_{x}\right)=\sigma(x)$. Note that for each $p \in Q, \pi^{-1}(p)$ is finite, as $\operatorname{HOM}\left(\Gamma, G_{x}\right)$ is finite for each $x \in \sigma^{-1}(p)$ and the action of an $h \in G_{1}$ with $t(h)=x$ identifies each element of $\operatorname{HOM}\left(\Gamma, G_{t(x)}\right)$ with an element of $\operatorname{HOM}\left(\Gamma, G_{x}\right)$.

Lemma 3.1. Suppose $Q$ is closed and $\Gamma$ is a finitely generated group. Then each $\Gamma$-sector is a closed orbifold, and the image of each $\Gamma$-sector under $\pi$ is a compact subset of $Q$.

Proof. Pick $(\phi) \in T_{Q}^{\Gamma}$. We first claim that $\pi\left(\tilde{Q}_{(\phi)}\right)$ is a compact subset of $Q$. For each $p \in Q$, pick a linear chart at $x \in G_{0}, \sigma(x)=p$, with image $U_{p} \subseteq Q$. For each chart with domain $V_{x}$, let $V_{x}^{\prime}$ be a $G_{x}$-invariant ball about $0 \in V_{x}$ such that $\overline{V_{x}^{\prime}} \subset V_{x}$. Let $U_{p}^{\prime}=\pi_{p}\left(V_{x}^{\prime}\right)$. Then the $U_{p}^{\prime}$ form an open cover of $Q$. As $Q$ is compact, there is a finite subcover $\left\{U_{p_{i}}^{\prime}\right\}$ for $i=1,2, \ldots, k$ covered by linear charts at points $x_{i}$ with $\sigma\left(x_{i}\right)=p_{i}$. Then

$$
\pi\left(\tilde{Q}_{(\phi)}\right)=\bigsqcup_{\left\{i: \exists \phi_{x_{i}} \in(\phi)\right\}} \pi_{x_{i}}\left(\overline{V_{x_{i}}^{\prime}} \cap V_{x_{i}}^{\left\langle\phi_{x_{i}}\right\rangle}\right),
$$

which is a finite union of closed sets and hence closed and compact.

Now, suppose $\left(\phi_{x}\right)_{i}$ is a sequence in $\tilde{Q}_{(\phi)}$. Then $\pi\left[\left(\phi_{x}\right)_{i}\right]=p_{i}$ is a sequence in the compact space $\pi\left(\tilde{Q}_{(\phi)}\right)$, implying that it has a subsequence $p_{i_{j}}$ with limit $p \in \pi\left(\tilde{Q}_{(\phi)}\right)$. As $\pi^{-1}(p) \cap \tilde{Q}_{(\phi)}$ is a finite set, there is at least one $\phi_{y} \in \pi^{-1}(p) \cap \tilde{Q}_{(\phi)}$ such that every neighborhood of $\phi_{y}$ contains an infinite number of the $\left(\phi_{x}\right)_{i_{j}}$. It follows that there is a subsequence of $\left(\phi_{x}\right)_{i_{j}}$ that converges to $\phi_{y}$, and that $\tilde{Q}_{(\phi)}$ is compact.

We define a relation on $T_{Q}^{\Gamma}$ as follows. We say that $(\psi) \leq(\phi)$, or equivalently $(\phi) \geq(\psi)$, if $\pi\left(\tilde{Q}_{(\psi)}\right) \subseteq \pi\left(\tilde{Q}_{(\phi)}\right)$, and we say that $(\phi) \equiv(\psi)$ if $\pi\left(\tilde{Q}_{(\phi)}\right)=$ $\pi\left(\tilde{Q}_{(\psi)}\right)$. It can happen that $(\phi) \equiv(\psi)$ with $(\phi) \neq(\psi)$. However, we can consider $\leq$ a partial order of the equivalence classes of $\Gamma$-sectors under the obvious equivalence relation $\equiv$. By $(\psi)<(\phi)$, then, we will mean that $(\psi) \leq(\phi)$ and $(\psi) \not \equiv(\phi)$.

The following technical lemma demonstrates that the partial order $\leq$ can be understood completely locally.

Lemma 3.2. Let $Q$ be an orbifold and $\Gamma$ a finitely generated group. Suppose there is an $x \in G_{0}$ and homomorphisms $\phi_{x}, \psi_{x}: \Gamma \rightarrow G_{x}$ in $\approx$-classes $(\phi)$ and $(\psi)$, respectively. Let $\left\{V_{x}, G_{x}, \pi_{x}\right\}$ be any linear chart for $Q$ at $x$.

(i) If $V_{x}^{\left\langle\psi_{x}\right\rangle} \subseteq V_{x}^{\left\langle\phi_{x}\right\rangle}$, then $(\psi) \leq(\phi)$.

(ii) If $V_{x}^{\left\langle\psi_{x}\right\rangle} \subset V_{x}^{\left\langle\phi_{x}\right\rangle}$, then $(\psi)<(\phi)$.

(iii) If $V_{x}^{\left\langle\psi_{x}\right\rangle}=V_{x}^{\left\langle\phi_{x}\right\rangle}$, then $(\psi) \equiv(\phi)$. 
Proof. First, we note that if any of the containment hypotheses involving $V^{\left\langle\psi_{x}\right\rangle}$ and $V^{\left\langle\phi_{x}\right\rangle}$ are true for any linear chart at $x$, then they are true for every linear chart at $x$. This follows from the fact that $G_{x}$ acts linearly in every such chart so that these spaces are subspaces of each $V_{x} \ni 0$; of course, subspaces are determined by their intersection with any neighborhood of the origin.

If $h \in G_{1}$ with $s(h)=x$, then $h$ defines an equivalent linear orbifold chart for $Q$ at $t(h)$ of the form $\left\{t \circ s_{h}^{-1}\left(V_{x}\right), h G_{x} h^{-1}, \pi_{t(h)}\right\}=\left\{V_{t(h)}, G_{t(h)}, \pi_{t(h)}\right\}$. It is clear that

$$
V_{x}^{\left\langle\psi_{x}\right\rangle} \subseteq V_{x}^{\left\langle\phi_{x}\right\rangle} \Longleftrightarrow V_{t(h)}^{\left\langle h \psi_{x}\right\rangle} \subseteq V_{t(h)}^{\left\langle h \phi_{x}\right\rangle}
$$

and

$$
V_{x}^{\left\langle\psi_{x}\right\rangle} \subset V_{x}^{\left\langle\phi_{x}\right\rangle} \Longleftrightarrow V_{t(h)}^{\left\langle h \psi_{x}\right\rangle} \subset V_{t(h)}^{\left\langle h \phi_{x}\right\rangle} .
$$

We begin by showing that the containment hypotheses are preserved by a local covering in either direction.

Suppose there is a $\psi_{y} \in \mathcal{S}_{\mathcal{G}}^{\Gamma}$ with $\psi_{x} \stackrel{\text { loc }}{\curvearrowright} \psi_{y}$; then, by Lemma 2.7. there is a linear chart $\left\{V_{x}, G_{x}, \pi_{x}\right\}$ at $x$ and an element of the orbit $\mathcal{G} \psi_{y}$ (which we assume, without loss of generality by equations (3.1) and (3.2), is equal to $\psi_{y}$ ) such that $y \in V_{x}$ and $\xi_{x}^{y} \circ \psi_{y}=\psi_{x}$. It follows, in particular, that $y \in V_{x}^{\left\langle\psi_{x}\right\rangle}$. Pick $\phi_{x}: \Gamma \rightarrow G_{x}$ and assume that $V_{x}^{\left\langle\psi_{x}\right\rangle} \subseteq V_{x}^{\left\langle\phi_{x}\right\rangle}$; then, as $y \in V_{x}^{\left\langle\phi_{x}\right\rangle}, \xi_{x}^{y}\left(G_{y}\right)$ contains $\operatorname{Im} \phi_{x}$ as a subgroup. Recalling that $\xi_{x}^{y}$ is injective, define

$$
\begin{aligned}
\phi_{y}: & \Gamma \longrightarrow G_{y} \\
: \gamma & \longmapsto\left(\xi_{x}^{y}\right)^{-1}\left[\phi_{x}(\gamma)\right] .
\end{aligned}
$$

Then $\phi_{y} \in \mathcal{S}_{\mathcal{G}}^{\Gamma}$. As $\xi_{x}^{y} \circ \phi_{y}=\phi_{x}$, it is clear that $\phi_{x} \stackrel{\text { loc }}{\curvearrowright} \phi_{y}$. Pick a linear chart $\left\{V_{y}, G_{y}, \pi_{y}\right\}$ at $y$, and assume by shrinking $V_{y}$ if necessary that $V_{y} \subseteq V_{x} \subseteq G_{0}$. Then it follows from the construction that $V_{y}^{\left\langle\phi_{y}\right\rangle}=V_{y} \cap V_{x}^{\left\langle\phi_{x}\right\rangle}$ and $V_{y}^{\left\langle\psi_{y}\right\rangle}=V_{y} \cap V_{x}^{\left\langle\psi_{x}\right\rangle}$. Therefore, $V_{y}^{\left\langle\psi_{y}\right\rangle} \subseteq V_{y}^{\left\langle\phi_{y}\right\rangle}$. Note further that if we assume a strict inclusion $V_{x}^{\left\langle\psi_{x}\right\rangle} \subset$ $V_{x}^{\left\langle\phi_{x}\right\rangle}$, then as both sets are subspaces of $V_{x}$, which is diffeomorphic to $\mathbb{R}^{n}$, and $V_{y}$ is an open subset of $V_{x}$, it follows that $V_{y}^{\left\langle\psi_{y}\right\rangle} \subset V_{y}^{\left\langle\phi_{y}\right\rangle}$ is strict.

On the other hand, if there is a $\psi_{y} \in \mathcal{S}_{\mathcal{G}}^{\Gamma}$ with $\psi_{y} \stackrel{\text { loc }}{\curvearrowright} \psi_{x}$, then by Lemma 2.7there is a linear chart $\left\{V_{y}, G_{y}, \pi_{y}\right\}$ at $y$ and a representative of $\mathcal{G} \psi_{x}$ (which we assume, again without loss of generality, is equal to $\psi_{x}$ ) such that $x \in V_{y}$ and $\xi_{y}^{x} \circ \psi_{x}=\psi_{y}$. Let $\left\{V_{x}, G_{x}, \pi_{x}\right\}$ be a linear chart at $x$; by shrinking $V_{x}$ if necessary, we assume that $V_{x} \subseteq V_{y} \subseteq G_{0}$. Assume that $V_{x}^{\left\langle\psi_{x}\right\rangle} \subseteq V_{x}^{\left\langle\phi_{x}\right\rangle}$ for some $\phi_{x}: \Gamma \rightarrow G_{x}$, and note that as $\xi_{y}^{x}: G_{x} \rightarrow G_{y}$ extends the $G_{x}$-action on $V_{x}$ to all of $V_{y}, V_{x}^{\left\langle\psi_{x}\right\rangle}=V_{x} \cap V_{y}^{\left\langle\psi_{y}\right\rangle}$. Defining $\phi_{x}=\xi_{y}^{x} \circ \phi_{y}$, we have that $\phi_{x} \in \mathcal{S}_{\mathcal{G}}^{\Gamma}$ and $\phi_{y} \stackrel{\text { loc }}{\curvearrowright} \phi_{x}$. As above, we have $V_{x}^{\left\langle\phi_{x}\right\rangle}=V_{x} \cap V_{y}^{\left\langle\phi_{y}\right\rangle}$, so that $V_{y}^{\left\langle\psi_{y}\right\rangle} \subseteq V_{y}^{\left\langle\phi_{y}\right\rangle}$. Again, as the sets in question are subspaces, if $V_{x}^{\left\langle\psi_{x}\right\rangle} \subset V_{x}^{\left\langle\phi_{x}\right\rangle}$, then $V_{y}^{\left\langle\psi_{y}\right\rangle} \subset V_{y}^{\left\langle\phi_{y}\right\rangle}$.

Now, suppose $V^{\left\langle\psi_{x}\right\rangle} \subseteq V^{\left\langle\phi_{x}\right\rangle}$. Let $q \in \pi\left(\tilde{Q}_{\left(\psi_{x}\right)}\right)$; then there is a $y \in G_{0}$ with $\sigma(y)=q$ and a $\psi_{y} \in \mathcal{S}_{\mathcal{G}}^{\Gamma}$ with $\psi_{y} \approx \psi_{x}$. By the definition of $\approx$, there is a finite sequence $\psi_{x_{0}}, \psi_{x_{1}}, \ldots, \psi_{x_{l}}$ such that $\psi_{x_{0}} \in \mathcal{G} \psi_{x}, \psi_{x_{l}} \in \mathcal{G} \psi_{y}$, and for each $i$, $\psi_{x_{i}} \stackrel{\text { loc }}{\curvearrowright} \psi_{x_{i+1}}$ or $\psi_{x_{i+1}} \stackrel{\text { loc }}{\curvearrowright} \psi_{x_{i}}$. By equation (3.1), we can assume that $\psi_{x_{0}}=\psi_{x}$ and $\psi_{x_{l}}=\psi_{y}$. Applying the above arguments for each $i$, we have that there is a 
sequence $\phi_{x_{0}}, \phi_{x_{1}}, \ldots, \phi_{x_{l}}$ such that $\phi_{x_{0}}=\phi_{x}, \phi_{x_{l}}=\phi_{y}$, and for each $i, \phi_{x_{i}} \stackrel{\text { loc }}{\curvearrowright} \phi_{x_{i+1}}$ or $\phi_{x_{i+1}} \stackrel{\text { loc }}{\curvearrowright} \phi_{x_{i}}$. At each step, $V^{\left\langle\psi_{x_{i}}\right\rangle} \subseteq V^{\left\langle\phi_{x_{i}}\right\rangle}$ implies that $V^{\left\langle\psi_{x_{i+1}}\right\rangle} \subseteq V^{\left\langle\phi_{x_{i+1}}\right\rangle}$. It follows that $q \in \pi\left(\tilde{Q}_{(\phi)}\right)$, proving $(i)$.

To prove (ii), we apply equation (3.2) and note that it has been shown above that $V^{\left\langle\psi_{x_{i}}\right\rangle} \subset V^{\left\langle\phi_{x_{i}}\right\rangle}$ implies $V^{\left\langle\psi_{x_{i+1}}\right\rangle} \subset V^{\left\langle\phi_{x_{i+1}}\right\rangle}$ for each $i$. To prove (iii), we simply apply $(i)$ to $\psi_{x}$ and $\phi_{x}$ and then reverse their roles.

Lemma 3.3. Let $Q$ be an orbifold and $\Gamma$ a finitely generated group. For each $(\phi) \in T_{Q}^{\Gamma}$, the set

$$
\pi\left(\tilde{Q}_{(\phi)}\right) \backslash \bigcup_{(\psi)<(\phi)} \pi\left(\tilde{Q}_{(\psi)}\right)
$$

is connected.

Proof. Pick an $x \in G_{0}$ and $\phi_{x} \in \mathcal{S}_{\mathcal{G}}^{\Gamma}$ such that $\phi_{x} \in(\phi)$. Then a chart for $\tilde{Q}_{(\phi)}$ at $\phi_{x}$ is of the form $\left\{V_{x}^{\left\langle\phi_{x}\right\rangle}, C_{G_{x}}\left(\phi_{x}\right), \pi_{x}^{\phi_{x}}\right\}$. By [13, Theorem 4.3.2, page 158], there is an open and dense subset $\mathcal{O}$ of $V_{x}^{\left\langle\phi_{x}\right\rangle}$ of principal orbit type with respect to the $C_{G_{x}}\left(\phi_{x}\right)$-action such that $\mathcal{O} / C_{G_{x}}\left(\phi_{x}\right)$ is connected.

If some point $y \in V_{x}^{\left\langle\phi_{x}\right\rangle}$ satisfies $\pi \circ \pi_{x}^{\phi_{x}}(y) \in \pi\left(\tilde{Q}_{(\psi)}\right)$ for some $(\psi)<(\phi)$, then there is a $\psi_{y} \in(\psi)$. This implies that the group $\left\langle\operatorname{Im} \psi_{y}, \operatorname{Im} \phi_{y}\right\rangle \leq G_{y}$ where $\phi_{y}=$ $\left(\xi_{x}^{y}\right)^{-1} \circ \phi_{x}$. It follows that $y$ is not an element of principal orbit type; otherwise, $\operatorname{Im}\left(\xi_{x}^{y} \circ \psi_{y}\right)$ would act trivially on $V_{x}^{\left\langle\phi_{x}\right\rangle}$, implying by Lemma 3.2 that $(\psi) \equiv(\phi)$ (contradicting the fact that $(\psi)<(\phi)$ ). Conversely, if $y$ is not of principal orbit type, then picking a surjective $\psi_{y}$ onto $G_{y}$ clearly defines a class $(\psi)$ with $(\psi)<(\phi)$. Hence, we have that in the image of each local chart, $\tilde{Q}_{(\phi)} \backslash \bigcup_{(\psi)<(\phi)} \pi^{-1} \circ \pi\left(\tilde{Q}_{(\psi)}\right)$ corresponds to the connected set of points with principal $C_{G_{x}}\left(\phi_{x}\right)$-orbit type. As $\tilde{Q}_{(\phi)}$ is connected, so that any two points can be connected by a path covered by such charts, this implies that $\tilde{Q}_{(\phi)} \backslash \bigcup_{(\psi)<(\phi)} \pi^{-1} \circ \pi\left(\tilde{Q}_{(\psi)}\right)$ is connected. Note that if $q \in \pi\left(\tilde{Q}_{(\psi)}\right)$ for some $(\psi)<(\phi)$, then the isotropy groups of the points in $\pi^{-1}(q) \cap \tilde{Q}_{(\phi)}$ are isomorphic. Therefore, each such point is contained in $\tilde{Q}_{\left(\psi^{\prime}\right)}$ for some $\left(\psi^{\prime}\right)<(\phi)$, and

$$
\pi\left(\tilde{Q}_{(\phi)} \backslash \bigcup_{(\psi)<(\phi)} \pi^{-1} \circ \pi\left(\tilde{Q}_{(\psi)}\right)\right)=\pi\left(\tilde{Q}_{(\phi)}\right) \backslash \bigcup_{(\psi)<(\phi)} \pi\left(\tilde{Q}_{(\psi)}\right)
$$

is the continuous image of a connected set, hence itself connected.

We note the following, which is a trivial consequence of Lemma 2.9.

Lemma 3.4. Let $Q$ be a closed orbifold and $\Gamma$ a finitely generated group. For each $\Gamma$-sector $\tilde{Q}_{(\phi)}$ of $Q$, there is a $\Gamma$-sector $\tilde{Q}_{(\psi)}$ of $Q$, with $(\psi) \leq(\phi)$, that represents a minimal $\equiv$-class with respect to $\leq$. In other words, if $\left(\phi^{\prime}\right) \leq(\psi)$, then $\left(\phi^{\prime}\right) \equiv(\psi)$.

We will abuse language slightly and say that $(\psi)$ is minimal with respect to $\leq$. By this we mean that the $\equiv$-class of $(\psi)$ is minimal. 
Definition 3.5 (Covering the local groups). We say that the group $\Gamma$ covers the local groups of $Q$ if for each subgroup $H$ of each isotropy group $G_{x}$ of $Q$, there is a homomorphism $\phi_{x}: \Gamma \rightarrow G_{x}$ with $\operatorname{Im} \phi_{x}=H$.

We note that for every compact orbifold $Q$, there is a finite group that covers the local groups of $Q$. See the proof of Lemma 2.9. for each $i=1,2, \ldots, k$, let $\left\{H_{i, j}: i=1,2, \ldots, l_{i}\right\}$ be a collection of all of the nontrivial subgroups of $G_{x_{i}}$. Then

$$
\bigoplus_{i=1}^{k} \bigoplus_{j=1}^{l_{i}} H_{i, j}
$$

covers the local groups of $Q$. Similarly, if $Q$ is any orbifold such that the number of generators in a presentation of an isotropy group of $Q$ is bounded by $d$, then the free group with $d$ generators $\mathbb{F}_{d}$ covers the local groups of $Q$.

Lemma 3.6. Suppose $\Gamma$ covers the local groups of $Q$ and $\pi\left(\tilde{Q}_{(\phi)}\right) \cap \pi\left(\tilde{Q}_{(\psi)}\right) \neq \emptyset$. Then there is a $\left(\psi^{\prime}\right) \in T_{Q}^{\Gamma}$ with $\left(\psi^{\prime}\right) \leq(\phi),\left(\psi^{\prime}\right) \leq(\psi)$, and $\pi\left(\tilde{Q}_{\left(\psi^{\prime}\right)}\right) \subseteq \pi\left(\tilde{Q}_{(\phi)}\right) \cap$ $\pi\left(\tilde{Q}_{(\psi)}\right)$. Moreover, $\pi\left(\tilde{Q}_{(\phi)}\right) \cap \pi\left(\tilde{Q}_{(\psi)}\right)$ is a union of the images of such sectors; i.e.

$$
\pi\left(\tilde{Q}_{(\phi)}\right) \cap \pi\left(\tilde{Q}_{(\psi)}\right)=\bigcup_{\left(\psi^{\prime}\right) \leq(\phi),\left(\psi^{\prime}\right) \leq(\psi)} \pi\left(\tilde{Q}_{\left(\psi^{\prime}\right)}\right) .
$$

Note that it is possible that $\left(\psi^{\prime}\right) \equiv(\phi),\left(\psi^{\prime}\right) \equiv(\psi)$, or both.

Proof. Pick $p \in \pi\left(\tilde{Q}_{(\phi)}\right) \cap \pi\left(\tilde{Q}_{(\psi)}\right)$ and $x \in G_{0}$ with $\sigma(x)=p$. Then there are $\phi_{x}, \psi_{x} \in \mathcal{S}_{\mathcal{G}}^{\Gamma}$ with $\phi_{x} \in(\phi), \psi_{x} \in(\psi)$. As $\Gamma$ covers the local groups of $Q$, let $\psi_{x}^{\prime}: \Gamma \rightarrow\left\langle\operatorname{Im} \phi_{x}, \operatorname{Im} \psi_{x}\right\rangle$ be surjective. Then clearly $V_{x}^{\left\langle\psi_{x}^{\prime}\right\rangle} \subseteq V_{x}^{\left\langle\phi_{x}\right\rangle}$ and $V_{x}^{\left\langle\psi_{x}^{\prime}\right\rangle} \subseteq$ $V_{x}^{\left\langle\psi_{x}\right\rangle}$. By Lemma 3.2, letting $\left(\psi^{\prime}\right)$ denote the $\approx$-class of $\psi_{x}^{\prime}$ as usual, $\left(\psi^{\prime}\right) \leq(\phi)$ and $\left(\psi^{\prime}\right) \leq(\psi)$.

This construction can be performed for each $p \in \pi\left(\tilde{Q}_{(\phi)}\right) \cap \pi\left(\tilde{Q}_{(\psi)}\right)$, so that any such $p$ is clearly contained in some $\pi\left(\tilde{Q}_{\left(\psi^{\prime}\right)}\right)$ with $\left(\psi^{\prime}\right) \leq(\phi),\left(\psi^{\prime}\right) \leq(\psi)$.

We note that in the case that $Q$ is an abelian orbifold, i.e. if each of the $G_{x}$ is abelian, then the restriction $\left.\pi\right|_{\tilde{Q}_{(\phi)}}$ of $\pi$ to any $\Gamma$-sector is injective and hence an embedding of $\tilde{Q}_{(\phi)}$ into $Q$ as a suborbifold. This follows from the fact that $C_{G\left(\phi_{x}\right)}=G_{x}$ for each $\phi_{x} \in \mathcal{S}_{\mathcal{G}}^{\Gamma}$. In general, however, $\left.\pi\right|_{\tilde{Q}_{(\phi)}}$ will fail to be injective. In Lemmas 3.7 and 3.8, we show that $\left.\pi\right|_{\tilde{Q}_{(\phi)}}$ is a sort of singular finite covering space, and its singularities occur precisely on the images of $\Gamma$-sectors $\tilde{Q}_{(\psi)}$ with $(\psi)<(\phi)$. When there are no such sectors, $\left.\pi\right|_{\tilde{Q}_{(\phi)}}$ is a covering space of smooth manifolds.

Lemma 3.7. Suppose $\Gamma$ covers the local groups of the orbifold $Q$. Let $\tilde{Q}_{(\phi)}$ have dimension $k$ and let $p \in \pi\left(\tilde{Q}_{(\phi)}\right)$. One of the following is true.

(i) The point $p$ is contained in $\pi\left(\tilde{Q}_{(\psi)}\right)$ for some $(\psi)<(\phi)$. 
(ii) There is a neighborhood $W$ of $p$ in $\pi\left(\tilde{Q}_{(\phi)}\right)$ which is diffeomorphic to $\mathbb{R}^{k}$ and such that $\pi^{-1}(W) \cap \tilde{Q}_{(\phi)}$ is a finite number of disjoint sets diffeomorphic to $W$.

In particular, the set $\pi\left(\tilde{Q}_{(\phi)}\right) \backslash \bigcup_{(\psi)<(\phi)} \pi\left(\tilde{Q}_{(\psi)}\right)$ is a smooth manifold equipped with the trivial action of a finite group.

Proof. Pick $p \in \pi\left(\tilde{Q}_{(\phi)}\right)$ and $x \in G_{0}$ with $\sigma(x)=p$. Let $\left\{V_{x}, G_{x}, \pi_{x}\right\}$ be a linear chart for $Q$ at $x$. Then as $p=\sigma(x) \in\left(\tilde{Q}_{(\phi)}\right)$, there is a $\phi_{x} \in \mathcal{S}_{\mathcal{G}}^{\Gamma}$ that is a representative of $(\phi)$. By Lemma 2.4 a linear chart at $\phi_{x}$ for the connected component $\tilde{Q}_{(\phi)}$ of $\tilde{Q}_{\Gamma}$ is $\left\{V_{x}^{\left\langle\phi_{x}\right\rangle}, C_{G_{x}}\left(\phi_{x}\right), \pi_{x}^{\phi_{x}}\right\}$. Note that $V_{x}^{\left\langle\phi_{x}\right\rangle}$ is a subspace of $V_{x}$, and as it forms an orbifold chart for $\tilde{Q}_{(\phi)}$, it has dimension $k$.

Suppose $G_{x}$ does not act trivially on $V_{x}^{\left\langle\phi_{x}\right\rangle}$ as a subset of $V_{x}$. This means that there is a $g \in G_{x}$ and a $y \in V_{x}^{\left\langle\phi_{x}\right\rangle}$ such that $t\left[\left(\xi_{x}^{y}\right)^{-1}(g)\right] \neq y$ (of course, $t\left[\left(\xi_{x}^{y}\right)^{-1}(g)\right]$ need not be an element of $\left.V_{x}^{\left\langle\phi_{x}\right\rangle}\right)$. As $\Gamma$ covers the local groups of $Q$, let $\psi_{x}: \Gamma \rightarrow G_{x}$ have image $\left\langle\operatorname{Im} \phi_{x}, g\right\rangle$. Then $V_{x}^{\left\langle\psi_{x}\right\rangle}$ is a proper subspace of $V_{x}^{\left\langle\phi_{x}\right\rangle}$ as it does not contain $y$. By Lemma 3.2. $\left(\psi_{x}\right)<\left(\phi_{x}\right)$, and $(i)$ is true.

Now, suppose $G_{x}$ acts trivially on $V_{x}^{\left\langle\phi_{x}\right\rangle}$. Then $\pi_{x}^{\phi_{x}}: V_{x}^{\left\langle\phi_{x}\right\rangle} \rightarrow \tilde{Q}_{(\phi)}$ is a diffeomorphism onto its image. Note that $\pi \circ \pi_{x}^{\phi_{x}}=\pi_{x}$ on $V_{x}^{\left\langle\phi_{x}\right\rangle}$ and that $\pi_{x}$ is the quotient map by the trivial $G_{x}$-action. Therefore, $\pi$ maps a neighborhood of $x$ diffeomorphic to $V_{x}^{\left\langle\phi_{x}\right\rangle}$ diffeomorphically onto a neighborhood of $p$ in $\pi\left(\tilde{Q}_{(\phi)}\right)$. Let $W=\pi_{x}\left(V_{x}^{\left\langle\phi_{x}\right\rangle}\right)$ be this neighborhood. Any other element of $\tilde{Q}_{(\phi)}$ in $\pi^{-1}(p)$ is of the form $h \phi_{x}$ for an $h \in G_{1}$ with $s(h)=x$. Then $\left\{V_{t(x)}^{\left\langle h \phi_{x}\right\rangle}, C_{G_{t(h)}}\left(h \phi_{x}\right), \pi_{t(h)}^{h \phi_{x}}\right\}$ is an equivalent orbifold chart for $\tilde{Q}_{(\phi)}$ at $h \phi_{x}$. Suppose $t(h)=x$ and $V_{t(x)}^{\left\langle h \phi_{x}\right\rangle} \neq V_{x}^{\left\langle\phi_{x}\right\rangle}$. As $\Gamma$ covers the local groups of $Q$, let $\psi_{x}: \Gamma \rightarrow G_{x}$ have image $\left\langle\operatorname{Im} \phi_{x}, \operatorname{Im} h \phi_{x}\right\rangle$. Then $V_{x}^{\left\langle\psi_{x}\right\rangle}=V_{t(x)}^{\left\langle h \phi_{x}\right\rangle} \cap V_{x}^{\left\langle\phi_{x}\right\rangle} \subset V_{x}^{\left\langle\phi_{x}\right\rangle}$ so that, by Lemma 3.2 $(\psi)<(\phi)$. Hence, if $t(h)=x$, then either $(i)$ is true or $V_{t(x)}^{\left\langle h \phi_{x}\right\rangle}=V_{x}^{\left\langle\phi_{x}\right\rangle}$.

If $t(h) \neq x$, then as the restriction $\left.\mathcal{G}\right|_{V_{x}}$ is isomorphic to $G_{x} \ltimes V_{x}, t(h) \notin V_{x}$. By shrinking $V_{x}$ (and hence $W$ ) if necessary, we may assume that as subsets of $G_{0}$, $V_{x}^{\left\langle\phi_{x}\right\rangle} \cap V_{t(h)}^{\left\langle h \phi_{x}\right\rangle}=\emptyset$. Therefore,

$$
\pi^{-1}(W) \cap \tilde{Q}_{(\phi)}=\bigsqcup_{h \in s^{-1}(x), t(h) \neq x} \pi_{t(h)}^{h \phi_{x}}\left(V_{t(h)}^{\left\langle h \phi_{x}\right\rangle}\right) .
$$

As $\pi^{-1}(p)$ and hence $\pi^{-1}(p) \cap \tilde{Q}_{(\phi)}$ is finite, the $\left\{V_{t(x)}^{\left\langle h \phi_{x}\right\rangle}, C_{G_{t(h)}}\left(h \phi_{x}\right), \pi_{t(h)}^{h \phi_{x}}\right\}$ form linear charts for a finite number of open subsets of $\tilde{Q}_{(\phi)}$. By the argument above, two sets $V_{t\left(h_{1}\right)}^{\left\langle h_{1} \phi_{x}\right\rangle}$ and $V_{t\left(h_{2}\right)}^{\left\langle h_{2} \phi_{x}\right\rangle}$ either are disjoint or coincide and yield equivalent linear charts. As each $V_{t(h)}^{\left\langle h \phi_{x}\right\rangle}$ is a $k$-dimensional subspace of $V_{t(h)}$ and $\pi_{t(h)}^{h \phi_{x}}$ is simply the quotient map of the trivial $C_{G_{t(h)}}\left(h \phi_{x}\right)$-action, (ii) is true. 
Now, for every point $p \in \pi\left(\tilde{Q}_{(\phi)}\right) \backslash \bigcup_{(\psi)<(\phi)} \pi\left(\tilde{Q}_{(\psi)}\right)$ there is a neighborhood $W_{p}$ of $p$ in $\pi\left(\tilde{Q}_{(\phi)}\right) \backslash \bigcup_{(\psi)<(\phi)} \pi\left(\tilde{Q}_{(\psi)}\right)$, diffeomorphic to $\mathbb{R}^{k}$, such that $\pi^{-1}\left(W_{p}\right) \cap \tilde{Q}_{(\phi)}$ is a finite number of sets diffeomorphic to $W_{p}$. For each $\mathcal{G} \phi_{x} \in \pi^{-1}(p) \cap \tilde{Q}_{(\phi)}$, there is a chart $\left\{V_{x}^{\left\langle\phi_{x}\right\rangle}, C_{G_{x}}\left(\phi_{x}\right), \pi_{x}^{\phi_{x}}\right\}$ for $\tilde{Q}_{(\phi)}$ at $\mathcal{G} \phi_{x}$ in which the preimage of $W_{p}$ corresponds to $V_{x}^{\left\langle\phi_{x}\right\rangle}$ with trivial $C_{G_{x}}\left(\phi_{x}\right)$-action. As $\tilde{Q}_{(\phi)}$ is connected, and as isotropy groups of objects in the same $\mathcal{G}$-orbit are isomorphic, it is easy to see that the isotropy group of each point in $\pi\left(\tilde{Q}_{(\phi)}\right) \backslash \bigcup_{(\psi)<(\phi)} \pi\left(\tilde{Q}_{(\psi)}\right)$ is isomorphic. Moreover, each of these manifold charts is the restriction of a linear orbifold chart to an invariant subspace. Hence, they patch together to give $\pi\left(\tilde{Q}_{(\phi)}\right) \backslash \underset{(\psi)<(\phi)}{\bigcup} \pi\left(\tilde{Q}_{(\psi)}\right)$ the structure of a smooth manifold.

Thus, $\pi\left(\tilde{Q}_{(\phi)}\right) \backslash \bigcup_{(\psi)<(\phi)} \pi\left(\tilde{Q}_{(\psi)}\right)$ is a smooth manifold equipped with the trivial action of a finite group; moreover, selecting $p \in \pi\left(\tilde{Q}_{(\phi)}\right) \backslash \bigcup_{(\psi)<(\phi)} \pi\left(\tilde{Q}_{(\psi)}\right)$ and $x \in G_{0}$ such that $\sigma(x)=p$, that finite group is given by $G_{x}$.

Lemma 3.8. Suppose $\Gamma$ covers the local groups of $Q$. If $(\phi)$ is a minimal element of $T_{Q}^{\Gamma}$, then both $\tilde{Q}_{(\phi)}$ and $\pi\left(\tilde{Q}_{(\phi)}\right)$ are smooth manifolds equipped with the trivial action of a finite group.

Proof. That $\pi\left(\tilde{Q}_{(\phi)}\right)$ is a manifold follows directly from Lemma 3.7. Clearly, in this case,

$$
\pi\left(\tilde{Q}_{(\phi)}\right) \backslash \bigcup_{(\psi)<(\phi)} \pi\left(\tilde{Q}_{(\psi)}\right)=\pi\left(\tilde{Q}_{(\phi)}\right) .
$$

That $\tilde{Q}_{(\phi)}$ is a manifold follows from the fact that the groups in the orbifold charts $\left\{V_{x}^{\left\langle\phi_{x}\right\rangle}, C_{G\left(\phi_{x}\right)}, \pi_{x}^{\phi_{x}}\right\}$ for $\tilde{Q}_{(\phi)}$ act trivially. Hence, $\tilde{Q}_{(\phi)}$ is an orbifold in which every element of the local group acts trivially; therefore the associated reduced orbifold is a smooth manifold.

Note in particular that if $(\phi)$ is minimal, then by Lemma 3.7. $\left.\pi\right|_{\tilde{Q}_{(\phi)}}$ is a covering map for its image.

\section{The Euler-Satake Characteristics and Classes AS COMPLETE OBSTRUCTIONS}

In this section, we use the constructions developed above to give a necessary and sufficient condition for an orbifold to admit a nonvanishing, smooth vector field. Our main result is Theorem 1.1 which deals with the case of a closed orbifold; this is proven in Subsection 4.1, In Subsection 4.2, we prove Theorem 4.7, dealing with the case of an orbifold with boundary, and Theorem 4.11, dealing with a certain class of open orbifolds.

We start with two lemmas dealing with continuously extending and smoothly approximating vector fields on closed orbifolds. 
Lemma 4.1. Let $Q$ be a closed orbifold and let $S \subseteq Q$ be closed. A continuous vector field on $S$ can be extended to a continuous vector field on $Q$.

Proof. Suppose $X_{0}$ is a vector field defined on $S$. Let $\left\{\left(U_{i}, f_{i}\right) \mid i=1,2, \ldots, m\right\}$ be a finite partition of unity for $Q$ composed of uniformized sets, each uniformized by a linear chart $\left\{V_{i}, G_{i}, \pi_{i}\right\}$. For each $i$ such that $S \cap U_{i}$ is not empty, we have that $\pi_{i}^{-1}(S)$ is a closed subset of $V_{i}$ and $\pi_{i}^{*} X_{0}$ is a $G_{i}$-invariant vector field on $\pi_{i}^{-1}(S)$. As $V_{i}$ is an open subset of $\mathbb{R}^{n}$, we can treat a vector field on $\pi_{i}^{-1}(S) \subseteq V_{i}$ as $n$ $\mathbb{R}^{n}$-valued functions $\left(\pi^{*} X_{0}\right)^{j}, j=1,2, \ldots, n$. Extending each of these functions to all of $V_{i}$ by the Tietsze Extension Theorem, we form a vector field $Y_{i}$ on $V_{i}$ that extends $\pi_{i}^{*} X_{0}$. Let $X_{i}$ be the average of $Y_{i}$ over the $G_{i}$-action, i.e.

$$
X_{i}=\frac{1}{\left|G_{i}\right|} \sum_{g \in G_{i}} g Y_{i}
$$

then as $\pi_{i}^{*} X_{0}$ is $G_{i}$-invariant, $X_{i}$ also extends $\pi_{i}^{*} X_{0}$.

For each $i$ such that $S \cap U_{i}=\emptyset$, let $Y_{i}$ be an arbitrary vector field on $V_{i}$ and let $X_{i}$ be its average over the $G_{i}$-action.

Since each $X_{i}$ is a $G_{i}$-invariant vector field on $V_{i}$, it defines a vector field on $U_{i}$ (also denoted by $\left.X_{i}\right)$. The vector field $X(p)=\sum_{i=1}^{m} f_{i}(p) X_{i}(p)$ is a continuous vector field on $Q$ that extends $X_{0}$.

Lemma 4.2. Let $Q$ be a closed orbifold that admits a continuous vector field that is nonvanishing on the closed set $S$. Then $Q$ admits a $\mathcal{C}^{\infty}$ vector field that is nonvanishing on the closed set $S$.

Proof. Let $Y$ be a continuous vector field on $Q$ that is nonvanishing on $S$. Fix a metric on $Q$ and let $M$ be the minimum value of $\|Y(p)\|$ on $S$. Then $M>0$.

For each $p \in Q$, pick a linear chart $\left\{V_{x}, G_{x}, \pi_{x}\right\}$ at some $x$ with $\sigma(x)=p$. Then $\pi_{x}^{*} Y$ is a continuous vector field so that there is a $G_{x}$-invariant open ball $W_{x}$ about $0 \in V_{x}$ such that

$$
\left\|\pi_{x}^{*} Y(y)-\pi_{x}^{*} Y(0)\right\|<\frac{M}{2} .
$$

Note that $\pi_{x}^{*} Y(0)$ is a $G_{x}$-invariant vector field. The collection of the $\pi_{x}\left(W_{x}\right)$ forms an open cover of $Q$, so let $\left\{\pi_{x_{i}}\left(W_{x_{i}}\right): i=1,2, \ldots, k\right\}$ be a finite subcover with $\sigma\left(x_{i}\right)=p_{i}$ for each $i$. Let $\left\{\rho_{i}: i=1,2, \ldots, k\right\}$ be a partition of unity subordinate to this subcover and define

$$
X(p)=\sum_{i=1}^{k} \rho_{i}(p) Y\left(p_{i}\right) .
$$

Note that $X(p)$ is a smooth vector field on $Q$. Then we have for each $p \in Q$ that

$$
\begin{aligned}
\|Y(p)-X(p)\| & =\left\|\sum_{i=1}^{k} \rho_{i}(p) Y(p)-\sum_{i=1}^{k} \rho_{i}(p) Y\left(p_{i}\right)\right\| \\
& =\left\|\sum_{\left\{i: p \in \operatorname{supp} \rho_{i}\right\}} \rho_{i}(p)\left(Y(p)-Y\left(p_{i}\right)\right)\right\| \\
& \leq \sum_{\left\{i: p \in \operatorname{supp} \rho_{i}\right\}} \rho_{i}(p)\left\|Y(p)-Y\left(p_{i}\right)\right\| \\
& <\sum_{\left\{i: p \in \operatorname{supp} \rho_{i}\right\}} \rho_{i}(p) \frac{M}{2} \\
& =\frac{M}{2} .
\end{aligned}
$$


Therefore,

$$
\begin{aligned}
\|X(p)\| & =\|Y(p)-(Y(p)-X(p))\| \\
& \geq\|Y(p)\|-\|Y(p)-X(p)\| \\
& >\|Y(p)\|-\frac{M}{2} \\
& >\frac{M}{2}>0 .
\end{aligned}
$$

Hence, $X$ is nonvanishing on $S$.

4.1. Closed orbifolds. We turn to the proof of Theorem 1.1. One direction of the theorem is true for any finitely generated group $\Gamma$, so we state and prove it as Lemma 4.3. To prove the other direction, we need to construct a smooth, nonvanishing vector field $X$ on a closed orbifold $Q$, assuming that $\Gamma$ covers the local groups of $Q$ and that $\chi_{E S}\left(\tilde{Q}_{(\phi)}\right)=0$ for each $\Gamma$-sector. We will construct $X$ on the $\Gamma$-sectors of $Q$ inductively using the partial order $\leq$. To simplify the exposition, we will organize this construction into two claims; Claim 4.4 is a base case and Claim 4.5 is the inductive step. The actual induction will be explained in the proof of the theorem.

Lemma 4.3. Let $Q$ be a closed orbifold and $\Gamma$ a finitely generated group. If $Q$ admits a nonvanishing, smooth vector field $X$, then $\chi_{E S}\left(\tilde{Q}_{(\phi)}\right)=0$ for each $(\phi) \in$ $T_{Q}^{\Gamma}$.

Proof. Suppose $Q$ admits a nonvanishing, smooth vector field $X$ and let $\tilde{X}_{\Gamma}$ denote the extension of $X$ to $\tilde{Q}_{\Gamma}$ defined in Lemma 2.11. Then $\tilde{X}_{\Gamma}$ is smooth and nonvanishing. Pick $(\phi) \in T_{Q}^{\Gamma}$. Letting $\operatorname{ind}^{\text {orb }}\left(\left.\tilde{X}_{\Gamma}\right|_{\tilde{Q}_{(\phi)}} ; \tilde{Q}_{(\phi)}\right)$ denote the index of the vector field $\left.\tilde{X}_{\Gamma}\right|_{\tilde{Q}_{(\phi)}}$ on the set $\tilde{Q}_{(\phi)}$ in the orbifold sense (see [15]), we have

$$
\operatorname{ind}^{\text {orb }}\left(\left.\tilde{X}_{\Gamma}\right|_{\tilde{Q}_{(\phi)}} ; \tilde{Q}_{(\phi)}\right)=0 .
$$

By the Poincaré-Hopf Theorem for closed orbifolds in [15], then,

$$
\begin{aligned}
0 & =\operatorname{ind}^{\text {orb }}\left(\left.\tilde{X}_{\Gamma}\right|_{\tilde{Q}_{(\phi)}} ; \tilde{Q}_{(\phi)}\right) \\
& =\chi_{E S}\left(\tilde{Q}_{(\phi)}\right) .
\end{aligned}
$$

Now, we assume that $\Gamma$ covers the local groups of the closed orbifold $Q$.

Claim 4.4 (Base case). Let $Q$ be a closed orbifold and $\Gamma$ a finitely generated group that covers the local groups of $Q$. If $\chi_{E S}\left(\tilde{Q}_{(\phi)}\right)=0$ for each minimal $(\phi) \in T_{Q}^{\Gamma}$, then there is a smooth vector field $X$ on $Q$ whose restriction to $\pi\left(\tilde{Q}_{(\phi)}\right)$ for each minimal $\Gamma$-sector $\tilde{Q}_{(\phi)}$ is nonvanishing.

Proof. Let $(\phi)$ be a minimal element of $T_{Q}^{\Gamma}$. Then $\tilde{Q}_{(\phi)}$ and $\pi\left(\tilde{Q}_{(\phi)}\right)$ are smooth manifolds equipped with the trivial action of a finite group, by Lemma 3.8. As all isotropy groups of $\tilde{Q}_{(\phi)}$ are isomorphic so that the Euler-Satake characteristic of $\tilde{Q}_{(\phi)}$ is simply its Euler characteristic divided by the order of any isotropy group, we see that $\chi_{\text {top }}\left(\tilde{Q}_{(\phi)}\right)=0$. Moreover, as $\left.\pi\right|_{\tilde{Q}_{(\phi)}}$ is a covering map onto $\pi\left(\tilde{Q}_{(\phi)}\right)$, it 
follows that $\chi_{t o p}\left(\pi\left(\tilde{Q}_{(\phi)}\right)\right)=0$. Hence, it admits a smooth, nonvanishing vector field.

Noting that the images of minimal $\Gamma$-sectors are either disjoint or coincide by Lemma 3.6. we can use this technique to construct a nonvanishing vector field on the image of each minimal $\Gamma$-sector in $Q$. By Lemma 3.1, the union of the images of the minimal sectors in $Q$ is a finite union of closed sets and hence closed. Therefore, by Lemma 4.1 we can extend to a vector field on all of $Q$ which, by Lemma 4.2 , we may assume is smooth.

Claim 4.5 (Induction step). Let $Q$ be a closed orbifold and $\Gamma$ a finitely generated group that covers the local groups of $Q$, and assume $\chi_{E S}\left(\tilde{Q}_{(\phi)}\right)=0$ for each $(\phi) \in T_{Q}^{\Gamma}$. Let $(\phi) \in T_{Q}^{\Gamma}$, and suppose there is a continuous vector field $X$ on $Q$ that restricts to a nonvanishing vector field on $\bigsqcup_{(\psi)<(\phi)} \pi\left(\tilde{Q}_{(\psi)}\right)$. Then there is a continuous vector field $Y$ on $Q$ that does not vanish on $\pi\left(\tilde{Q}_{(\phi)}\right)$ and coincides with $X$ on each $\pi\left(\tilde{Q}_{(\psi)}\right)$ such that $(\psi)<(\phi)$.

Proof. The zeros of $\left.X\right|_{\pi\left(\tilde{Q}_{(\phi)}\right)}$ are contained in $\pi\left(\tilde{Q}_{(\phi)}\right) \backslash \bigcup_{(\psi)<(\phi)} \pi\left(\tilde{Q}_{(\psi)}\right)$. By Lemmas 3.3 and 3.7, this set is a connected manifold. As in Lemma 3.7, fix a point $p \in \pi\left(\tilde{Q}_{(\phi)}\right) \backslash \bigcup_{(\psi)<(\phi)} \pi\left(\tilde{Q}_{(\psi)}\right)$ and an open neighborhood $W$ of $p$. We may assume, by shrinking $W$ if necessary, that $W$ is contained in the open set $\pi\left(\tilde{Q}_{(\phi)}\right) \backslash \bigcup_{(\psi)<(\phi)} \pi\left(\tilde{Q}_{(\psi)}\right)$. As $X$ does not vanish on the closed set $\underset{(\psi)<(\phi)}{\bigsqcup} \pi\left(\tilde{Q}_{(\psi)}\right)$, we may continuously perturb $\left.X\right|_{\pi\left(\tilde{Q}_{(\phi)}\right)}$ away from each of the $\pi\left(\tilde{Q}_{(\psi)}\right)$, and so we can assume that the zeros of $X$ are isolated and contained in the interior of a compact set $K \subset W$.

We have that $\pi^{-1}(K) \cap \tilde{Q}_{(\phi)}$ is a finite disjoint union of sets diffeomorphic to $K$, say $\pi^{-1}(K) \cap \tilde{Q}_{(\phi)}=\bigsqcup_{j=1}^{l} J_{i}$ where each $J_{i}$ is diffeomorphic to $K$. Hence, $\tilde{X}_{\Gamma}$ restricts to a continuous vector field $\left.\tilde{X}_{\Gamma}\right|_{\tilde{Q}_{(\phi)}}$ on $\tilde{Q}_{(\phi)}$ with only isolated zeros contained in the $J_{i}$ such that each of the $\left.\tilde{X}_{\Gamma}\right|_{J_{i}}$ coincides with $\left.X\right|_{K}$ via the diffeomorphism between each $J_{i}$ and $K$. We have by the Poincaré-Hopf Theorem for closed orbifolds in [15] that

$$
\begin{aligned}
0 & =\chi_{E S}\left(\tilde{Q}_{(\phi)}\right) \\
& =\operatorname{ind}^{\text {orb }}\left(\tilde{X}_{\Gamma} ; \tilde{Q}_{(\phi)}\right) \\
& =\sum_{i=1}^{l} \operatorname{ind}^{\text {orb }}\left(\tilde{X}_{\Gamma} ; J_{i}\right) \\
& =l\left(\operatorname{ind}^{\text {orb }}\left(\left.X\right|_{\pi\left(\tilde{Q}_{(\phi)}\right)} ; K\right)\right),
\end{aligned}
$$

so that ind ${ }^{\text {orb }}\left(\left.X\right|_{\pi\left(\tilde{Q}_{(\phi)}\right)} ; K\right)=0$. By techniques in [6], $\left.X\right|_{\pi\left(\tilde{Q}_{(\phi)}\right)}$ can be perturbed continuously on an open set whose closure is contained in $K$, resulting in a continuous, nonvanishing vector field on $\pi\left(\tilde{Q}_{(\phi)}\right)$. Applying Lemma 4.1 with 
$S=\pi\left(\tilde{Q}_{(\phi)}\right)$, we can extend to a continuous vector field $Y$ on $Q$ that does not vanish on $\pi\left(\tilde{Q}_{(\phi)}\right)$ and coincides with $X$ on each $\pi\left(\tilde{Q}_{(\psi)}\right)$ with $(\psi)<(\phi)$.

Proof of Theorem 1.1. Let $Q$ be a closed orbifold and $\Gamma$ a finitely generated group that covers the local groups of $Q$. Note that if $Q$ is oriented, then $\chi_{E S}\left(\tilde{Q}_{(\phi)}\right)=0$ for each $(\phi) \in T_{Q}^{\Gamma}$ is equivalent to $e_{\Gamma}^{E S}(Q)=0$ by Lemma 2.15, Let $T_{\Gamma, 0}^{Q} \subseteq T_{Q}^{\Gamma}$ denote the minimal elements. Let $T_{\Gamma, 1}^{Q}$ denote the set of $(\phi) \in T_{Q}^{\Gamma} \backslash T_{\Gamma, 0}^{Q}$ such that whenever $(\psi)<(\phi),(\psi) \in T_{\Gamma, 0}^{Q}$. Similarly, for each natural $j$, let $T_{\Gamma, j}^{Q}$ denote the set of $(\phi) \in T_{Q}^{\Gamma} \backslash \bigcup_{i=0}^{j-1} T_{\Gamma, i}^{Q}$ such that whenever $(\psi)<(\phi),(\psi) \in T_{\Gamma, i}^{Q}$ for some $i<j$. Then by Lemma 2.9, there is an $m$ such that

$$
T_{Q}^{\Gamma}=\bigcup_{i=1}^{m} T_{\Gamma, i}^{Q} .
$$

In particular, (1) $\in T_{\Gamma, m}^{Q}$ where (1) denotes the $\approx$-class of the trivial homomorphism into any isotropy group.

By Claim 4.4, there is a smooth vector field $X_{0}$ on $Q$ that does not vanish on $\bigsqcup_{(\phi) \in T_{\Gamma, 0}^{Q}} \pi\left(\tilde{Q}_{(\phi)}\right)$. Pick $j$ with $1 \leq j<m$ and assume that there is a continuous vector field $Y_{j}$ on $Q$ that is nonvanishing on $\pi\left(\tilde{Q}_{(\psi)}\right)$ for each $(\psi) \in T_{\Gamma, j}^{Q}$. This implies that $Y_{j}$ is nonvanishing on $\pi\left(\tilde{Q}_{(\psi)}\right)$ for each $(\psi) \in T_{\Gamma, i}^{Q}$ with $i \leq j$.

For each $(\phi) \in T_{\Gamma, j+1}^{Q}$, by Claim 4.5 we can construct a continuous vector field $Y_{(\phi)}$ on $Q$ such that the restriction $\left.Y_{(\phi)}\right|_{\pi\left(\tilde{Q}_{(\phi)}\right)}$ to $\pi\left(\tilde{Q}_{(\phi)}\right)$ is nonvanishing. If $(\phi),\left(\phi^{\prime}\right) \in T_{\Gamma, j+1}^{Q}$, then $Y_{(\phi)}$ and $Y_{\left(\phi^{\prime}\right)}$ need not coincide. However, since they both extend $Y_{j}$, it is clear that they coincide on $\pi\left(\tilde{Q}_{(\psi)}\right)$ for each $(\psi) \in T_{\Gamma, i}^{Q}$ with $i \leq j$. Moreover, by Lemma 3.6] $\pi\left(\tilde{Q}_{(\phi)}\right) \cap \pi\left(\tilde{Q}_{\left(\phi^{\prime}\right)}\right)$ is a union of such $\pi\left(\tilde{Q}_{(\psi)}\right)$, so that $Y_{(\phi)}$ and $Y_{\left(\phi^{\prime}\right)}$ coincide on $\pi\left(\tilde{Q}_{(\phi)}\right) \cap \pi\left(\tilde{Q}_{\left(\phi^{\prime}\right)}\right)$. Hence, if we set

$$
Y_{j+1}(p)=Y_{(\phi)}(p) \quad \forall p \in \pi\left(\tilde{Q}_{(\phi)}\right),(\phi) \in T_{\Gamma, j+1}^{Q},
$$

then $Y_{j+1}$ will be a well-defined, continuous, and nonvanishing vector field on $\bigcup_{(\phi) \in T_{\Gamma, j+1}^{Q}} \pi\left(\tilde{Q}_{(\phi)}\right)$. As this set is closed, we apply Lemma 4.1 to extend $Y_{j+1}$ to a continuous vector field (also denoted by $Y_{j+1}$ ) on $Q$ that is nonvanishing on $\pi\left(\tilde{Q}_{(\phi)}\right)$ for each $(\phi) \in T_{\Gamma, j+1}^{Q}$.

By induction, then, there is a continuous, nonvanishing vector field $Y_{m}$ on $\tilde{Q}_{(1)}$, which is diffeomorphic to $Q$. By Lemma 4.2, we can approximate $Y_{m}$ with a smooth, nonvanishing vector field, completing the proof of Theorem 1.1 .

We end this subsection with an example of a closed orbifold that does not admit a nonvanishing vector field. In this case, the obstruction is not detected when $\Gamma=\mathbb{Z}$ yet is detected for other choices of $\Gamma$. 
Example 4.6. Let $\mathbb{R}^{6}$ have standard basis $\left\{e_{1}, e_{2}, e_{3}, e_{4}, e_{5}, e_{6}\right\}$, and let the dihedral group $D_{6}$ act on the sphere $S^{5} \subset \mathbb{R}^{6}$ as follows. We let $a$ denote the permutation (123) acting on the basis elements and let

$$
b=\left[\begin{array}{cccccc}
0 & 1 & 0 & 0 & 0 & 0 \\
1 & 0 & 0 & 0 & 0 & 0 \\
0 & 0 & 1 & 0 & 0 & 0 \\
0 & 0 & 0 & -1 & 0 & 0 \\
0 & 0 & 0 & 0 & 1 & 0 \\
0 & 0 & 0 & 0 & 0 & 1
\end{array}\right]
$$

act by a permutation along with multiplying the fourth basis element by -1 . One checks that $\langle a, b\rangle$ is isomorphic to the dihedral group $D_{6}$. We let $Q=S^{5} / D_{6}$; then the orbifold groupoid $\mathcal{G}=S^{5} \ltimes D_{6}$ is a representative of the orbifold structure of $Q$.

Letting $\Gamma=\mathbb{Z}$ with generator $\gamma$, there are three $\Gamma$-sectors. The first corresponds to the homomorphism $\gamma \mapsto 1$ at each point, and is clearly diffeomorphic to $Q$. The second corresponds to $\gamma \mapsto a$ over points stabilized by $a$, and is given by $S^{3}=$ Span $\left\{e_{1}+e_{2}+e_{3}, e_{4}, e_{5}, e_{6}\right\} \cap S^{5}$ with trivial $\mathbb{Z}_{3}$-action. The third corresponds to $\gamma \mapsto b$ over points stabilized by $b$, and is given by $S^{3}=\operatorname{Span}\left\{e_{1}+e_{2}, e_{3}, e_{5}, e_{6}\right\} \cap S^{5}$ with trivial $\mathbb{Z}_{2}$-action. We note that the $\mathbb{Z}$-sectors correspond to the inertia orbifold; the Euler-Satake characteristics of each of these sectors is equal to zero, as they are all odd-dimensional orbifolds (see [15, Theorem 4]).

Now, let $\Gamma=\mathbb{F}_{2}$, the free group with generators $\gamma_{1}$ and $\gamma_{2}$. Designating a homomorphism $\mathbb{F}_{2} \rightarrow D_{6}$ by $\left(g_{1}, g_{2}\right)$ where $\gamma_{i} \mapsto g_{i}$, we have the following conjugacy classes:

$$
\{(1,1)\},
$$

mapping into the isotropy group over every point;

$$
\begin{aligned}
& \left\{(1, a),\left(1, a^{2}\right)\right\}, \\
& \left\{(a, 1),\left(a^{2}, 1\right)\right\} \text {, } \\
& \left\{(a, a),\left(a^{2}, a^{2}\right)\right\} \text {, } \\
& \left\{\left(a, a^{2}\right),\left(a^{2}, a\right)\right\} \text {, }
\end{aligned}
$$

mapping into the isotropy group over every point in $S^{3}=\operatorname{Span}\left\{e_{1}+e_{2}+e_{3}, e_{4}, e_{5}, e_{6}\right\}$ $\cap S^{5}$;

$$
\begin{gathered}
\left\{(1, b),(1, a b),\left(1, a^{2} b\right)\right\}, \\
\left\{(b, 1),(a b, 1),\left(a^{2} b, 1\right)\right\}, \\
\left\{(b, b),(a b, a b),\left(a^{2} b, a^{2} b\right)\right\},
\end{gathered}
$$

mapping into the isotropy group over every point in $S^{3}=\operatorname{Span}\left\{e_{1}+e_{2}, e_{3}, e_{5}, e_{6}\right\} \cap$ $S^{5}$ (or a representative of the orbit of this set); and

$$
\begin{gathered}
\left\{(b, a b),\left(a b, a^{2} b\right),\left(a^{2} b, b\right),(a b, b),\left(a^{2} b, a b\right),\left(b, a^{2} b\right)\right\}, \\
\quad\left\{(a, b),(a, a b),\left(a, a^{2} b\right),\left(a^{2}, b\right),\left(a^{2}, a b\right),\left(a^{2}, a^{2} b\right)\right\}, \\
\left\{(b, a),(a b, a),\left(a^{2} b, a\right),\left(b, a^{2}\right),\left(a b, a^{2}\right),\left(a^{2} b, a^{2}\right)\right\},
\end{gathered}
$$

mapping into the isotropy group over every point in $S^{2}=\operatorname{Span}\left\{e_{1}+e_{2}+e_{3}, e_{5}, e_{6}\right\} \cap$ $S^{5}$.

The first conjugacy class corresponds to a sector diffeomorphic to $Q$. The next four correspond to sectors diffeomorphic to $S^{3} \bmod$ the trivial action of $\mathbb{Z}_{3}$, already represented by the $\mathbb{Z}$-sectors. The following three correspond to sectors diffeomorphic to $S^{3} \bmod$ the trivial action of $\mathbb{Z}_{2}$, also diffeomorphic to a $\mathbb{Z}$-sector. The final 
three conjugacy classes, however, comprise points with isotropy $D_{6}$ with only the trivial group acting. These sectors are given by $S^{2}=\operatorname{Span}\left\{e_{1}+e_{2}+e_{3}, e_{5}, e_{6}\right\} \cap S^{5}$ and have nonzero Euler-Satake characteristic. Note that the $\mathbb{F}_{2}$-sectors correspond to the space of 2-multisectors (see [1, page 54]) and that $\mathbb{F}_{2}$ covers the local groups of $Q$.

We see, then, that although the $\mathbb{Z}$-sectors do not detect any obstruction to the existence of nonvanishing vector fields, the $\mathbb{F}_{2}$-sectors do. We note that the obstruction is also detected using $\Gamma=D_{6}$ and $\Gamma=\mathbb{Z}_{2} \oplus \mathbb{Z}_{3} \oplus D_{6}$; the latter of these covers the local groups, while the former does not.

4.2. Compact orbifolds with boundary and open suborbifolds of closed orbifolds. Although it is likely that the construction of the $\Gamma$-sectors extends naturally to the case of an orbifold with boundary (whose orbifold structure is given by a Lie groupoid with $G_{0}$ and $G_{1}$ being manifolds with boundary), we will not develop the construction in this case. Rather, we will use the double orbifold to define them.

Let $Q$ be a compact $n$-dimensional orbifold with boundary (see [3] or [16] for the definition). Form the double $\widehat{Q}$ (see [12, Section 3]) and let $\widehat{\mathcal{G}}$ be an orbifold groupoid for $\widehat{Q}$ with objects $\widehat{G}_{0}$, arrows $\widehat{G}_{1}$, source $\widehat{s}$, target $\widehat{t}$, quotient projection $\widehat{\sigma}$, etc. Form the $\Gamma$-sectors $(\widehat{Q})_{\Gamma}$. Treating $Q$ as a subset of $\widehat{Q}$, we let

$$
\tilde{Q}_{\Gamma}=\widetilde{(\widehat{Q}}_{\Gamma} \cap \widehat{\pi}^{-1}(Q)
$$

Then if $\left\{V_{x}, G_{x}, \pi_{x}\right\}$ is a linear chart at $x \in \widehat{G}_{0}$ such that $\widehat{\sigma}(x)=p \in \partial Q \subseteq$ $Q \subset \widehat{Q}$, a chart for $Q$ can be taken to be $\left\{V_{x}^{+}, G_{x}, \pi_{x}\right\}$ where $V_{x}^{+}$is an open subset of $\mathbb{R}_{+}^{n}=\left\{\left(x_{1}, \ldots, x_{n}\right): x_{n} \geq 0\right\}$. For each $\phi_{x} \in \widehat{\pi}^{-1}(p)$, a neighborhood of $\phi_{x} \in \tilde{Q}_{\Gamma}$ is covered by the chart with boundary $\left\{V_{x}^{\left\langle\phi_{x}\right\rangle} \cap V_{x}^{+}, C_{G_{x}}\left(\phi_{x}\right), \pi_{x}^{\phi_{x}}\right\}=$ $\left\{\left(V_{x}^{+}\right)^{\left\langle\phi_{x}\right\rangle}, C_{G_{x}}\left(\phi_{x}\right), \pi_{x}^{\phi_{x}}\right\}$. Hence, we see that $\tilde{Q}_{\Gamma}$ has the structure of an orbifold with boundary and $\left(\widehat{\widehat{Q})_{\Gamma}}\right.$ is the double orbifold of $\tilde{Q}_{\Gamma}$.

It is easy to see that each $(\phi) \in T_{\widehat{Q}}^{\Gamma}$ has a representative $\phi_{x}$ with $\widehat{\pi}\left(\phi_{x}\right) \in Q$, so we define $T_{Q}^{\Gamma}=T_{\widehat{Q}}^{\Gamma}$. For each $(\phi) \in T_{Q}^{\Gamma}$, let

$$
\tilde{Q}_{(\phi)}=\widetilde{(\widehat{Q})}_{(\phi)} \cap \widehat{\pi}^{-1}(Q)
$$

Then we clearly have

$$
\tilde{Q}_{\Gamma}=\bigsqcup_{(\phi) \in T_{Q}^{\Gamma}} \tilde{Q}_{(\phi)}
$$

Let $\pi: \tilde{Q}_{\Gamma} \rightarrow Q$ be defined as the restriction of $\widehat{\pi}$ to $\tilde{Q}_{\Gamma} \subset \widetilde{(\widehat{Q})_{\Gamma}}$, and note that the relation $\leq$ defined on $T_{\widehat{Q}}^{\Gamma}$ coincides with its natural definition on $T_{Q}^{\Gamma}$. In other words, $(\psi) \leq(\phi)$ as elements of $T_{\widehat{Q}}^{\Gamma}$ if and only if $\pi\left(\tilde{Q}_{(\psi)}\right) \subseteq\left(\tilde{Q}_{(\phi)}\right)$.

We also define

$$
\overline{T_{Q}^{\Gamma}}=\left\{(\phi) \in T_{Q}^{\Gamma}: \partial\left(\tilde{Q}_{(\phi)}\right)=\emptyset\right\}
$$

to be the set of all $\Gamma$ sectors of $Q$ that are closed orbifolds. Note that $\Gamma$ covers the local groups of $Q$ if and only if $\Gamma$ covers the local groups of $\widehat{Q}$. 
Theorem 4.7. Let $Q$ be a compact orbifold with boundary. Let $\Gamma$ be a finitely generated group that covers the local groups of $Q$. Then $Q$ admits a smooth nonvanishing vector field if and only if $\chi_{E S}\left(\tilde{Q}_{(\phi)}\right)=0$ for each $(\phi) \in \overline{T_{Q}^{\Gamma}}$.

Again, we note that no requirement is made of the behavior of the vector field on the boundary of $Q$.

The proof of this theorem is similar to that of Theorem 1.1. The primary difference is the observation that a vector field need not vanish on $\widehat{\pi}\left({\widetilde{(\widehat{Q})_{(\phi)}}}_{(\text {for some }}\right.$ $(\phi) \in T_{Q}^{\Gamma} \backslash \bar{T}_{Q}^{\Gamma}$. However, since the images of these sectors intersect the boundary, zeros can be "pushed off" to occur outside of $Q$ in $\widehat{Q}$.

Lemma 4.8. Let $Q$ be a compact orbifold with boundary. If $Q$ admits a nonvanishing, smooth vector field $X$, then for every finitely generated $\Gamma, \chi_{E S}\left(\tilde{Q}_{(\phi)}\right)=0$ for each $(\phi) \in \bar{T}_{Q}^{\Gamma}$.

Proof. Suppose $Q$ admits a nonvanishing, smooth vector field $X$. As $Q$ is closed in $\widehat{Q}$, we can extend $X$ to a vector field $\widehat{X}$ by Lemma 4.1 which, by Lemma 4.2 we can assume is smooth. By Lemma 2.11, $\widehat{X}$ induces a vector field on $\widetilde{(\widehat{Q})_{\Gamma}}$; let $\tilde{X}_{\Gamma}$ denote the restriction of this vector field to $\tilde{Q}_{\Gamma}$. Then $\tilde{X}_{\Gamma}$ is smooth and nonvanishing. For each $(\phi) \in \bar{T}_{Q}^{\Gamma}$, we have that $\tilde{Q}_{(\phi)}$ is a closed orbifold and $\tilde{X}_{\Gamma}$ is a smooth, nonvanishing vector field on $\tilde{Q}_{(\phi)}$. Therefore, by the Poincaré-Hopf Theorem for closed orbifolds in [15], $\chi_{E S}\left(\tilde{Q}_{(\phi)}\right)=0$.

Now assume $\Gamma$ covers the local groups of $Q$.

Claim 4.9 (Base Case). Let $Q$ be a compact orbifold with boundary and $\Gamma$ a finitely generated group that covers the local groups of $Q$. If $\chi_{E S}\left(\tilde{Q}_{(\phi)}\right)=0$ for each $(\phi) \in \bar{T}_{Q}^{\Gamma}$, then there is a smooth vector field $X$ on $Q$ whose restriction to $\pi\left(\tilde{Q}_{(\phi)}\right)$ for each minimal $(\phi) \in T_{Q}^{\Gamma}$ is nonvanishing.

Proof. Let $(\phi)$ be a minimal element of $T_{Q}^{\Gamma}$. If $(\phi) \in \bar{T}_{Q}^{\Gamma}$, then as $(\phi)$ is a minimal element of $T_{\widehat{Q}}^{\Gamma}$ and $\chi_{E S}\left(\tilde{Q}_{(\phi)}\right)=0$, the same technique used in the proof of Claim 4.4 can be used to construct a nonvanishing, smooth vector field $X_{(\phi)}$ on $\pi\left(\tilde{Q}_{(\phi)}\right)$. If $(\phi) \notin \bar{T}_{Q}^{\Gamma}$, then $\widehat{\pi}\left(\widetilde{(\widehat{Q})_{(\phi)}}\right)$ is a closed manifold by Lemma 3.8, Let $\widehat{X}_{(\phi)}$ be a smooth vector field on $\widehat{\pi}\left(\overline{(\widehat{Q})_{(\phi)}}\right)$ with isolated zeros. For each zero of $\widehat{X}_{(\phi)}$ in $p \in \pi\left(\tilde{Q}_{(\phi)}\right)$, pick a simple smooth curve $c(t)$ in $\widehat{\pi}\left(\widetilde{(\widehat{Q})_{(\phi)}}\right)$ with $c(0)=p$ and $c(1) \in \widehat{Q} \backslash Q$. Given a tubular neighborhood $W$ of the image of $c$, the vector field $\widehat{X}_{(\phi)}$ can be smoothly perturbed on a compact subset of $W$ so that it vanishes only at $c(1)$. Applying this to each zero in $\pi\left(\tilde{Q}_{(\phi)}\right)$ and then letting $X_{(\phi)}$ be the 
restriction of $\widehat{X}_{(\phi)}$ to $\pi\left(\tilde{Q}_{(\phi)}\right)$, we can assume $X_{(\phi)}$ is a nonvanishing vector field on $\pi\left(\tilde{Q}_{(\phi)}\right)$.

Again, the images of minimal $\Gamma$-sectors are either disjoint or coincide by Lemma 3.6. so we can construct a nonvanishing vector field on the image of each minimal $\Gamma$-sector in $Q$. By Lemma 4.1, as the union of the images of the minimal sectors in $Q$ is closed in $\widehat{Q}$, we can extend to a vector field $\widehat{X}$ on all of $\widehat{Q}$ which, by Lemma 4.2. we may assume is smooth. Then the required $X$ is the restriction of $\widehat{X}$ to $Q$.

Claim 4.10 (Induction Step). Let $Q$ be a compact orbifold with boundary and $\Gamma$ a finitely generated group that covers the local groups of $Q$. Suppose $\chi_{E S}\left(\tilde{Q}_{(\phi)}\right)=0$ for each $(\phi) \in \bar{T}_{Q}^{\Gamma}$. Let $(\phi) \in T_{Q}^{\Gamma}$, and suppose there is a continuous vector field $X$ on $Q$ that restricts to a nonvanishing vector field on $\bigsqcup_{(\psi)<(\phi)} \pi\left(\tilde{Q}_{(\psi)}\right)$. Then there is a continuous vector field $Y$ on $Q$ that does not vanish on $\pi\left(\tilde{Q}_{(\phi)}\right)$ and coincides with $X$ on each $\pi\left(\tilde{Q}_{(\psi)}\right)$ with $(\psi)<(\phi)$.

Proof. If $(\phi) \in \bar{T}_{Q}^{\Gamma}$, then $\pi\left(\tilde{Q}_{(\phi)}\right)$ is closed, so the proof is identical to that of Claim 4.5. On the other hand, if $(\phi) \notin \bar{T}_{Q}^{\Gamma}$, then the set $\pi\left(\widetilde{(\widehat{Q})_{(\phi)}}\right) \backslash \underset{(\psi)<(\phi)}{\bigcup} \pi\left(\widetilde{(\widehat{Q})_{(\psi)}}\right)$ is a manifold. Therefore, $\left.X\right|_{\pi\left(\widetilde{\left.(\widehat{Q})_{(\phi)}\right)}\right.}$ can be continuously perturbed away from each of the $\pi\left(\widetilde{(\widehat{Q})_{(\psi)}}\right)$ with $(\psi)<(\phi)$ so that it vanishes only on $\widehat{Q} \backslash Q$. The resulting vector field can be extended by Lemma 4.1 to a continuous vector field $\widehat{Y}$ on $\widehat{Q}$; the required vector field $Y$ is the restriction $\widehat{Y}$ to $Q$.

Proof of Theorem 4.7. Given Claims 4.9 and 4.10, the proof of Theorem 4.7 is identical to the proof of Theorem 1.1 .

Techniques almost identical to those above can be used to prove the following.

Theorem 4.11. Let $Q$ be an open suborbifold of the closed orbifold $R$. Let $\Gamma$ be a finitely generated group that covers the local groups of $Q$. Then $Q$ admits a smooth nonvanishing vector field if and only if $\chi_{E S}\left(\tilde{Q}_{(\phi)}\right)=0$ for each $(\phi) \in T_{Q}^{\Gamma}$ such that $\tilde{Q}_{\left(\phi_{x}\right)}$ is a closed orbifold.

For this case, we note that each $(\phi) \in T_{Q}^{\Gamma}$ determines an $\approx$-class in $T_{R}^{\Gamma}$. The correspondence $(\phi) \underset{\approx}{\approx} \mapsto(\phi)_{\approx}^{R}$ is neither surjective nor injective. Rather than use this correspondence, we apply the induction in the proof of Theorem 1.1 to the sectors of $T_{R}^{\Gamma}$, using the techniques in the proof of Theorem 4.7 when $\pi\left(\tilde{Q}_{(\phi)}\right)$ is not completely contained in $R$, i.e. when $\tilde{Q}_{(\phi)}$ is not closed. With this observation, the argument extends directly. 


\section{REFERENCES}

1. A. Adem, J. Leida, and Y. Ruan. Orbifolds and stringy topology, Cambridge Tracts in Mathematics 171, Cambridge University Press, Cambridge, 2007. MR 2359514

2. J. Bryan and J. Fulman, Orbifold Euler characteristics and the number of commuting m-tuples in the symmetric groups, Ann. Comb. 2 (1998) 1-6. MR.1682916 (2000f:20002)

3. W. Chen and Y. Ruan, Orbifold Gromov-Witten theory, in: Orbifolds in mathematics and physics (Madison, WI, 2001), Contemp. Math., 310, Amer. Math. Soc., Providence, RI, 2002, pp. 25-85. MR1950941 (2004k:53145)

4. W. Chen and Y. Ruan, A new cohomology theory of orbifold, Commun. Math. Phys. 248 (2004) 1-31. MR2104605 (2005j:57036)

5. M. Crainic and I. Moerdijk, Foliation groupoids and their cyclic homology, Adv. Math. 157 (2001) 177-197. MR 1813430(2002a:22004)

6. V. Guillemin and A. Pollack, Differential topology, Prentice-Hall, Inc., Englewood Cliffs, New Jersey, 1965. MR0348781 (50:1276)

7. J. Leida, Orbifolds and stable homotopy groups, preprint. arXiv:math/0505431v1 [math.AT] (2005).

8. I. Moerdijk, Orbifolds as groupoids: an introduction, in: Orbifolds in mathematics and physics (Madison, WI, 2001), Contemp. Math., 310, Amer. Math. Soc., Providence, RI, 2002, pp. 205222. MR.1950948 (2004c:22003)

9. I. Moerdijk and D.A. Pronk, Orbifolds, sheaves and groupoids, K-theory 12 (1997) 3-21. MR,1466622 (98i:22004)

10. I. Moerdijk and D. A. Pronk, Simplicial cohomology of orbifolds, Indag. Math. (N.S.) 10 (1999) 269-293. MR.1816220 (2002b:55012)

11. T. Ohmoto, Generating functions of orbifold Chern classes I: Symmetric products, Math. Proc. Cambridge Philos. Soc. 144 (2008) 423-438. MR 2405899 (2009e:14008)

12. E. Paquette and C. Seaton, The index of a vector field on an orbifold with boundary, Involve 2 (2009) 161-175.

13. M. Pflaum, Analytic and geometric study of stratified spaces, Lecture Notes in Mathematics 1768, Springer-Verlag, Berlin, 2001. MR.1869601 (2002m:58007)

14. Y. Ruan, Stringy geometry and topology of orbifolds, in: Symposium in honor of C. H. Clemens (Salt Lake City, UT, 2000), Contemp. Math., 312, Amer. Math. Soc., Providence, RI, 2002, pp. 187-233. MR.1941583 (2004b:32051)

15. I. Satake, The Gauss-Bonnet theorem for V-manifolds Journ. Math. Soc. Japan 9 (1957) 464-492. MR0095520 (20:2022)

16. C. Seaton, Two Gauss-Bonnet and Poincaré-Hopf theorems for orbifolds with boundary, Differential Geom. Appl. 26 (2008) 42-51. MR.2393971

17. C. Seaton, A complete obstruction to the existence of nonvanishing vector fields on almostcomplex, closed, cyclic orbifolds, preprint. arXiv:math/0408187v3 [math.DG] (2006).

18. C. Seaton, Characteristic classes of bad orbifold vector bundles, J. Geom. Phys. 57 (2007) 2365-2371. MR2360246

19. H. Tamanoi, Generalized orbifold Euler characteristic of symmetric products and equivariant Morava K-theory, Algebr. Geom. Topol. 1 (2001) 115-141. MR.1805937 (2002e:57052)

20. H. Tamanoi, Generalized orbifold Euler characteristic of symmetric orbifolds and covering spaces, Algebr. Geom. Topol. 3 (2003) 791-856. MR.1997338(2005j:57025)

Department of Mathematics, University of Colorado at Boulder, Campus Box 395, Boulder, Colorado 80309-0395

E-mail address: farsi@euclid.colorado.edu

Department of Mathematics and Computer Science, Rhodes College, 2000 N. ParkWAy, Memphis, Tennessee 38112

E-mail address: seatonc@rhodes.edu 